\title{
Calibration of Strapdown Magnetometers in the Magnetic Field Domain
}

\author{
Demoz Gebre-Egziabher*, Gabriel H. Elkaim, J. David Powell ${ }^{\ddagger}$ and Bradford W. Parkinson ${ }^{\S}$
}

\begin{abstract}
This paper presents an algorithm for calibrating strapdown magnetometers in the magnetic field domain. In contrast to the traditional method of compass swinging, which computes a series of heading correction parameters and, thus, is limited to use with two-axis systems, this algorithm estimates magnetometer output errors directly. Therefore, this new algorithm can be used to calibrate a full three-axis magnetometer triad. The calibration algorithm uses an iterated, batch least squares estimator which is initialized using a novel two-step nonlinear estimator. The algorithm is simulated to validate convergence characteristics and further validated on experimental data collected using a magnetometer triad. It is shown that the post calibration residuals are small and result in a system with heading errors on the order of 1 to 2 degrees.
\end{abstract}

\section{INTRODUCTION}

Magnetometers measure the intensity of magnetic fields and are used in many scientific and engineering applications. In vehicle navigation, for example, they are used as inexpensive heading sensors

\footnotetext{
*Assistant Professor, Department of Aerospace Engineering and Mechanics, University of Minnesota, Twin Cities, 110 Union St., N.E., 107 Akerman Hall, Minneapolis, MN, 55455. gebre@aem.umn.edu

${ }^{\dagger}$ Assistant Professor, Computer Engineering Department, University of California, Santa Cruz, 1156 High Street, Santa Cruz, CA 95064. elkaim@soe.ucsc.edu

${ }^{\ddagger}$ Professor Emeritus, Department of Aeronautics and Astronautics, Stanford University, Durand Building, 496 Lomita Mall, Stanford, CA 94305-4035. JDPowell@stanford.edu

$\S$ Professor Emeritus, Department of Aeronautics and Astronautics, Stanford University, Durand Building, 496 Lomita Mall, Stanford, CA 94305-4035. brad@relgyro.stanford.edu
} 
(where heading is the angle between the vehicle and North). In these applications, heading is determined by measuring the horizontal component of Earth's magnetic field vector, $\vec{h}$, using a perpendicular pair (or an orthogonal triad) of magnetometers. A magnetometer triad would measure $\vec{h}^{b}=\left[\begin{array}{lll}h_{x}^{b} & h_{y}^{b} & h_{z}^{b}\end{array}\right]^{T}$ which, as indicated by the superscript $b$, is Earth's magnetic field vector $\vec{h}$ expressed in a coordinate frame fixed to the body of the vehicle. A pair of magnetometers would measure only two components of the field vector or $\vec{h}^{b}=\left[\begin{array}{ll}h_{x}^{b} & h_{y}^{b}\end{array}\right]^{T}$.

If the the $x-y$ plane of the body coordinate system is level (i.e., is parallel to Earth's local tangent), the magnetometer readings are used to compute heading with respect to magnetic North using the following formula:

$$
\psi=-\tan ^{-1}\left(\frac{h_{y}^{b}}{h_{x}^{b}}\right)
$$

If the $x-y$ plane is not level, it can be levelled analytically by measuring the pitch and roll angles of the vehicle. These angles are used to compute the body-to-locally-level transformation matrix, $\stackrel{b \rightarrow w}{C}$, which is used to map the magnetometer measurement to the locally-level plane. Note that $\stackrel{b \rightarrow w}{C}$ is the " $b$ " to " $w$ " coordinate frame transformation matrix where the " $w$ " is the $x-y-z$ coordinate frame having its $x$ axis coincident with the vehicle's longitudinal axis and its $x$-y plane level or parallel to the local tangent (i.e., the wander azimuth plane).

Since the output of any sensor is, to some degree, corrupted by errors, the actual measurement made by the pair or triad of magnetometers, $\hat{\vec{h}}^{b}$, will be different from the true magnetic field $\vec{h}^{b}$. The process of estimating these errors and removing them from the magnetometer measurements is the subject of this paper.

For heading determination systems which use a pair of perpendicular magnetometers, a well known calibration procedure called compass swinging has been used successfully $[1,2]$. Compass swinging has several shortcomings, however, which make it unsuitable for many current applications. Two of most significant shortcomings of this procedure are that (1) it requires an external or independent source of heading and (2) the plane containing the pair of magnetometers must be level. These two factors limit or even preclude in-situ calibration because the magnetometer being calibrated are normally the 
only source of heading information. Furthermore, the " $b$ " and " $w$ " coordinate frames will not always be coincident.

Another deficiency of compass swinging is that it is a heading domain calibration algorithm. That is, it involves computing a series of heading correction parameters which, when added to heading (as computed by Equation 1), cancel heading deviations caused by magnetometer errors. As such, the algorithm is not applicable for applications other than heading determination (e.g., three axis attitude determination as discussed in [3], [4] and [5]). Another shortcoming of compass swinging is the fact that the quality of the calibration degrades as the vehicle with the magnetometers are moved farther away from the geographical point where the calibration was performed. This is because the correction parameters computed are functions of the local magnetic field strength.

Recently, different magnetometer calibration methodologies that deal with some of the shortcoming of compass swinging have been proposed. For example, [6] proposed a method which computes correction parameters for the magnetometer's field measurement errors instead of heading correction parameters. Since this is a procedure for calibration in the magnetic field domain, unlike compass swinging, the results are location independent. In addition, the calibration is not limited to magnetometers used solely for heading determination but applicable in cases where the magnetometers are used for any purpose [3]). However, the method discussed in [6] does have some limitations. For example, it still requires that the $x-y$ body plane of the vehicle containing the magnetometers be level during calibration which severely limits the algorithm from real time use in a vehicle that is moving.

In order to improve the performance the above discussed magnetic field domain calibration procedure, this paper presents a reformulation and extension of the method discussed in [6]. Section 2 presents a unified error model for strapdown magnetometers and discusses the problem of calibrating strapdown magnetometers in the context of the unified error model. In Section 3, prior art in calibration of magnetometers is presented. In particular, we will discuss compass swinging or the classical method of calibrating magnetometers in the heading domain. This will serve as a motivation for the methods presented in this paper as well as being a benchmark against which we will compare these new calibration methods. Section 4 will develop an iterative, batch least squares algorithm for calibration in 
the magnetic field domain. We will also present a two step, non-linear estimator used to establish the initial conditions for the iterative, batch least squares algorithm. In Section 5, the results of simulation and trade studies will be presented. Experimental results are presented in Section 6 and Section 7 will conclude this paper.

\section{ERROR MODELLING}

The output of magnetometers are corrupted by wide band measurement noise, stochastic biases due to sensor imperfections, installation errors and unwanted magnetic interference in the vicinity of the sensors.

The unwanted or interfering magnetic fields can be classified into two distinct groups. The first group consists of constant or slowly time-varying fields generated by ferromagnetic structural materials in the proximity of the magnetometers. The field measurement errors resulting from such interferences are referred to as hard iron biases [7].

The second group of interfering magnetic fields result from materials that generate their own magnetic field in response to an externally applied field. This generated field is affected by both the magnitude and direction of the externally applied magnetic field. Such materials are called soft irons and the error they generate is referred to as a soft iron bias. In a moving vehicle, the orientation of Earth's magnetic field relative to the vehicle (and any soft iron materials contained therein) changes continuously. Thus, the resulting soft iron errors are time varying.

A comprehensive mathematical model for the output error of a strapdown magnetometer can be written as:

$$
\hat{\vec{h}}^{w}=\stackrel{b \rightarrow w}{C}\left[C_{m} C_{s f} C_{s i}\left(\vec{h}^{b}+\vec{b}^{b}+\vec{w}^{b}\right)\right] .
$$

In this model, $\vec{b}^{b}=\left[\begin{array}{lll}b_{x}^{b} & b_{y}^{b} & b_{z}^{b}\end{array}\right]^{T}$ and $\vec{w}^{b}=\left[\begin{array}{lll}w_{x}^{b} & w_{y}^{b} & w_{z}^{b}\end{array}\right]^{T}$ represent hard iron biases and wideband noise, respectively, and are additive errors which corrupt the true field measurement $\vec{h}^{b}$. The variables $C_{s i}, C_{s f}$ and $C_{m}$ are $3 \times 3$ matrices that account for soft iron, scale factor and misalignment errors, respectively. The matrix $\stackrel{b \rightarrow w}{C}$ is the $3 \times 3$ body-to-local tangent transformation matrix discussed 
earlier. These errors are discussed in more detail next.

\subsection{Hard Iron Errors $\left(\vec{b}^{b}\right)$}

Normally, the largest errors tend to be null-shifts caused by unwanted magnetic fields in the vicinity of the magnetometers. This can be seen clearly by examining Figure 1 which shows the output error from a magnetometer measuring the vertical component of Earth's magnetic field vector, $h_{z}^{b}$, at Stanford, California (approximately $N 37.5^{\circ}$ latitude and $W 122.1^{\circ}$ longitude). Earth's magnetic field vector at

this location, $\vec{h}$, in North-East-Down (NED) coordinates is equal to $\left[\begin{array}{lll}0.23199 & 0.06361 & 0.43500\end{array}\right]^{T}$ Gauss [8]. Figure 1 shows the output of a magnetometer measuring the vertical component after $h_{z}$ (0.43500 Gauss) has been subtracted. What is shown in the figure, therefore, is a null-shift of 9.2 milliGauss and the largest component of the output error.

\subsection{Wide Band Noise $\left(\sigma_{w}\right)$}

Removing the null-shift from the data shown in Figure 1 leaves errors due to magnetometer measurement wide band noise. Figure 2 is a histogram of the data shown in Figure 1. From Figure 2 it can be seen that the wide band noise has a standard deviation, $\sigma_{w}$, of approximately 5 milli-Gauss which is smaller than the null shift.

\subsection{Soft Iron Errors $\left(C_{s i}\right)$}

In this work we assume that a linear relationship exits between the field a soft iron generates in response to an externally applied field. Even though this is a reasonable assumption in many applications, there are cases where it is invalid due to hysteresis. Assuming no hysteresis, $C_{s i}$ can be written as:

$$
C_{s i}=\left[\begin{array}{ccc}
\alpha_{x x} & \alpha_{x y} & \alpha_{x z} \\
\alpha_{y x} & \alpha_{y y} & \alpha_{y z} \\
\alpha_{z x} & \alpha_{z y} & \alpha_{z z}
\end{array}\right]
$$


The $\alpha_{i j}$ terms represent the effective soft iron coefficients. They are the constants of proportionality between the magnetic field applied to a soft iron and the resulting induced magnetic field. From a notation point of view, $\alpha_{x y}$, for example, represents the effective coefficient relating the field generated in the x-direction in response to an applied field in the y-direction. The term "effective" is used to describe these coefficients because they represent the effect of all soft iron material present that may corrupt the magnetometer outputs $[4,5]$.

\subsection{Scale Factor $\left(C_{s f}\right)$}

Scale factor errors are modeled using the $3 \times 3$ matrix $C_{s f}$ given by:

$$
C_{s f}=\left[\begin{array}{ccc}
\left(1+s_{f x}\right) & 0 & 0 \\
0 & \left(1+s_{f y}\right) & 0 \\
0 & 0 & \left(1+s_{f z}\right)
\end{array}\right]
$$

The scale factor errors $s_{f x}, s_{f y}$ and $s_{f z}$ represent the uncertainty in knowledge of the constant of proportionality relating magnetometer input to output.

\subsection{Misalignments $\left(C_{m}\right)$}

In an ideal installation, the magnetometer triad will be mounted in perfect alignment with the body axis of the aircraft. Stated differently, the magnetometer axes will be identical to the body axes. In actual practice, perfect alignment cannot always be achieved. The matrix $C_{m}$ accounts for this misalignment

and is nothing more than the magnetometer axes to body frame direction cosine matrix, ${ }^{p \rightarrow b}$. Since the misalignment between the two axes is normally very small (but not negligible), $C_{m}$ can be modeled as the following skew-symmetric matrix:

$$
C_{m}=\left[\begin{array}{ccc}
1 & -\epsilon_{z} & \epsilon_{y} \\
\epsilon_{z} & 1 & -\epsilon_{x} \\
-\epsilon_{y} & \epsilon_{x} & 1
\end{array}\right]
$$


The three independent parameters defining the matrix $\left(\epsilon_{x}, \epsilon_{y}\right.$ and $\left.\epsilon_{x}\right)$ represent small rotations about the body axes of the vehicle that will bring the platform axes into perfect alignment with the body axes. Thus, $C_{m}$ is constant and only needs to be estimated once.

The process of calibrating a pair or triad of magnetometers involves estimating the various unknown vectors and matrices defined in Equations 2 through 5. Methods for estimating these unknown parameters is the subject of the remainder of this paper.

\section{COMPASS SWINGING}

The compass swinging algorithm has been used for a some time in marine [1] and aviation [2] applications. In these applications, the sensors traditionally used were a pair of flux-gate or flux-valve magnetometers. The sensors are arranged perpendicular to each other and coincident with the $x$ and $y$ body axes of the vehicle. For these systems, the heading error, $\delta \psi$, due to both hard and soft iron biases is given by:

$$
\delta \psi=A+B \sin (\psi)+C \cos (\psi)+D \sin (2 \psi)+E \cos (2 \psi) .
$$

This equation is derived in the appendix at the end of this paper and from that derivation it can be seen that the coefficients $A$ through $E$ are functions of the soft iron coupling terms, $\alpha_{i j}$, and Earth's local horizontal magnetic field strength, $\vec{h}_{h}$.

The unknown coefficients $A$ through $E$ are estimated by levelling and rotating the vehicle through a series of $N$ known headings as shown schematically in Figure 3. At each known $k^{\text {th }}$ heading, the heading error, $\delta \psi_{k}$, is computed and used to form the following system of equations:

$$
\left[\begin{array}{c}
\delta \psi_{1} \\
\delta \psi_{2} \\
\vdots \\
\delta \psi_{N}
\end{array}\right]=\left[\begin{array}{ccccc}
1 & \sin \left(\psi_{1}\right) & \cos \left(\psi_{1}\right) & \sin \left(2 \psi_{1}\right) & \cos \left(2 \psi_{1}\right) \\
1 & \sin \left(\psi_{2}\right) & \cos \left(\psi_{2}\right) & \sin \left(2 \psi_{2}\right) & \cos \left(2 \psi_{2}\right) \\
\vdots & & \ddots & & \vdots \\
1 & \sin \left(\psi_{N}\right) & \cos \left(\psi_{N}\right) & \sin \left(2 \psi_{N}\right) & \cos \left(2 \psi_{N}\right)
\end{array}\right]\left[\begin{array}{c}
A \\
B \\
C \\
D \\
E
\end{array}\right]
$$


A batch least squares solution of Equation 7 yields estimates for the coefficients A through E.

Examination of Equations 6 and 7 reveals at least two short comings of the compass swinging procedure. First, the fact that the coefficients $A$ through $E$ are functions of $\vec{h}_{h}$ (see Appendix A) implies that the calibration is location dependent. Thus, if the vehicle is expected to travel over a large distances, multiple calibrations must be performed due to variations in Earth's magnetic field.

The second shortcoming of compass swinging becomes apparent when we note that heading is a required input to the algorithm. Since heading errors due to hard and soft iron errors are heading dependent, the heading input into the algorithm will be corrupted by a non-constant bias. Thus, an independent measurement of heading is required when calibrating magnetometers using this method. In aviation applications, the standard practice is to use a compass rose painted on the tarmac similar to what is shown in Figure 3, as the secondary independent heading measurement.

\section{CALIBRATION ALGORITHM}

In addition to the above noted limitations, the fact that the vehicle containing the magnetometers has to be level during calibration prevents use of compass swinging in motion. To deal with these shortcoming, an alternative calibration algorithm has been developed. The new algorithm works with both a pair or triad of magnetometers. We first develop the algorithm assuming only a pair of magnetometers are used. Once the basic algorithm has been developed, it is extended to three dimensions such that it is applicable to magnetometer triads. The method developed, corrects the field measurement errors directly and not the effect of field measurement errors on heading. Thus, as noted earlier, we will refer to this as a method of calibration in the magnetic field domain.

The fundamental idea behind calibration in the magnetic field domain is the fact that the locus of error-free measurements from a pair of perpendicular magnetometers is a circle. It is easy to show that this is the case by examining the expressions for $h_{x}^{b}$ and $h_{y}^{b}$. From Figure 3 it is clear to see that $h_{x}^{b}=h_{h} \cos (\psi)$ and $h_{y}^{b}=-h_{h} \sin (\psi)$. Squaring these expressions and adding them together leads to 
the following equation:

$$
\left(h_{x}^{b}\right)^{2}+\left(h_{y}^{b}\right)^{2}=h_{h}^{2} \cos ^{2} \psi+h_{h}^{2} \sin ^{2} \psi=h_{h}^{2}
$$

This is the equation of a circle with its center at the origin. The radius of the circle is equal to the magnitude of the horizontal component of the local Earth magnetic field vector which is a function of geographical location. The magnitude of the radius varies with latitude, longitude and altitude because Earth's magnetic field vector varies with location. This variation of Earth's magnetic field vector can be modeled with reasonable accuracy using the current International Geomagnetic Reference Field model (IGRF) [8].

The effect of the various magnetometer errors described in Equation 2 is to alter the shape of the locus of measurements described by Equation 8. In this instance, as noted in Equation 2, the erroneous $x$ and $y$ magnetometer outputs will be $\hat{h}_{x}^{b}$ and $\hat{h}_{y}^{b}$. Hard iron errors, for example, shift the origin of the basic locus. This can be shown mathematically by considering a hard iron bias vector with components $b_{x}$ and $b_{y}$. If the $x$ and $y$ field measurements in the platform axes are biased by $b_{x}$ and $b_{y}$, respectively, the equation for the locus of the magnetometer measurements becomes:

$$
\left(\hat{h}_{x}^{b}-b_{x}\right)^{2}+\left(\hat{h}_{y}^{b}-b_{y}\right)^{2}=h_{h}^{2}
$$

This is still the equation of a circle but instead of having its center located at the origin, its center is at $\left(b_{x}, b_{y}\right)$. In the absence of other forms of errors, scale factor errors cause the body $\mathrm{x}$ and $\mathrm{y}$ magnetometer measurements to be different when both are subjected to an identical magnetic field. This can be expressed mathematically as follows:

$$
\begin{aligned}
& \hat{h}_{x}^{b}=\left(1+s_{f x}\right) h_{h} \cos \psi, \\
& \hat{h}_{y}^{b}=-\left(1+s_{f y}\right) h_{h} \sin \psi .
\end{aligned}
$$


Squaring Equations 10 and 11 and adding them together leads to

$$
\left(\frac{\hat{h}_{x}^{b}}{1+s_{f x}}\right)^{2}+\left(\frac{\hat{h}_{y}^{b}}{1+s_{f y}}\right)^{2}=h_{h}{ }^{2},
$$

which is the equation of an ellipse centered at the origin. The major and minor axes' magnitudes are determined by the scale factor errors, $s_{f x}$ and $s_{f y}$. When the hard iron erros $b_{x}$ and $b_{y}$ are included in Equations 10 and 11, the resulting locus is still an ellipse but its center is moved away from the origin to $\left(b_{x}, b_{y}\right)$. That is,

$$
\left(\frac{\hat{h}_{x}^{b}-b_{x}}{1+s_{f x}}\right)^{2}+\left(\frac{\hat{h}_{y}^{b}-b_{y}}{1+s_{f y}}\right)^{2}=h_{h}{ }^{2} .
$$

Soft iron errors will modify the error-free circular locus into an ellipse but also rotate the major and minor axes of the ellipse. To show this mathematically, consider expressions for $\hat{h}_{x}^{b}$ and $\hat{h}_{y}^{b}$ when soft iron biases are the only sources of error. In this instance $\hat{h}_{x}^{b}$ and $\hat{h}_{y}^{b}$ become (see Equations 55 and 56 of Appendix A):

$$
\begin{aligned}
& \hat{h}_{x}^{b}=h_{h} \cos \psi\left(1+\alpha_{x x}\right)-\alpha_{x y} h_{h} \sin \psi \\
& \hat{h}_{y}^{b}=-h_{h} \sin \psi\left(1+\alpha_{y y}\right)-\alpha_{y x} h_{h} \cos \psi .
\end{aligned}
$$

In matrix form these equations become:

$$
\left[\begin{array}{c}
\hat{h}_{x}^{b} \\
\hat{h}_{y}^{b}
\end{array}\right]=\left[\begin{array}{cc}
\left(1+\alpha_{x x}\right) & \alpha_{x y} \\
\alpha_{y x} & \left(1+\alpha_{y y}\right)
\end{array}\right]\left[\begin{array}{c}
h_{h} \cos \psi \\
-h_{h} \sin \psi
\end{array}\right]
$$

Inverting this matrix equation and noting that $h_{x}^{b}=h_{h} \cos (\psi)$ and $h_{y}^{b}=-h_{h} \sin (\psi)$ leads to the following:

$$
\left[\begin{array}{c}
h_{x}^{b} \\
h_{y}^{b}
\end{array}\right]=\frac{1}{\left(1+\alpha_{x x}\right)\left(1+\alpha_{y y}\right)-\alpha_{x y} \alpha_{y x}}\left[\begin{array}{cc}
\left(1+\alpha_{y y}\right) & -\alpha_{x y} \\
-\alpha_{y x} & \left(1+\alpha_{x x}\right)
\end{array}\right]\left[\begin{array}{c}
\hat{h}_{x}^{b} \\
\hat{h}_{y}^{b}
\end{array}\right]
$$

If the two equations represented by this matrix are squared and added, the resulting locus will describe 
an ellipse with rotated major and minor axes. If, in addition to soft iron errors, hard iron errors are present, the locus will still be a rotated ellipse but its center will be displaced from the origin. Figure 4 is a graphical summary of the effect of the various errors on the locus of magnetometer measurements. For the remainder of this paper, we will assume that $C_{m}$ and $\alpha_{i j}$ where $i \neq j$ are zero. This is not always a reasonable assumption. However, for the experimental work that will be discussed later in the paper, $C_{m}$ was made as small as possible by careful installation of the magnetometers and accounting for the residuals in post process. Furthermore, examination of experimental data indicated that the ellipse (or ellipsoid in the three dimensional case) had major and minor axes aligned with the body axes justifying the assumption that $\alpha_{i j}=0$ when $i \neq j$. With these simplifications, the hard iron errors affect the center of the elliptical measurement locus while the scale factor errors and the $\alpha_{i i}$ soft iron terms affect the size of the major and minor axes.

The calibration algorithm that will be developed is nothing more than a parameter estimation problem. The algorithm is an attempt to fit the best ellipse (in the least squares sense) to the measured magnetometer data. In the case of a magnetometer triad, the error-free locus of outputs is a sphere. This sphere will be centered at the origin with a radius equal to the magnitude of Earth's magnetic field vector.

The various magnetometer errors alter the spherical locus into an ellipse and displace it from the center. In particular, the scale factor and $\alpha_{i i}$ soft iron terms reshape the sphere into an ellipsoid centered at the origin. Hard iron errors shift the ellipsoid away from the origin and the effect of the wide-band noise is to roughen the smooth surface of the measurement locus.

Thus, the calibration algorithm is the problem of determining the parameters of an ellipsoid that best fit the data collected from a magnetometer triad. Mathematically, the locus of measurements is described by the following equation:

$$
\|\vec{h}\|^{2}=h^{2}=\left(\frac{\hat{h}_{x}^{b}-b_{x}}{\gamma_{x}}\right)^{2}+\left(\frac{\hat{h}_{y}^{b}-b_{y}}{\gamma_{y}}\right)^{2}+\left(\frac{\hat{h}_{z}^{b}-b_{z}}{\gamma_{z}}\right)^{2}
$$


where,

$$
\begin{aligned}
& \gamma_{x}=\left(1+s_{f x}\right)\left(1+\alpha_{x x}\right), \\
& \gamma_{y}=\left(1+s_{f y}\right)\left(1+\alpha_{y y}\right), \\
& \gamma_{z}=\left(1+s_{f z}\right)\left(1+\alpha_{z z}\right) .
\end{aligned}
$$

The parameters to be estimated are the hard iron biases denoted by $b_{x}, b_{y}, b_{z}$ and the combined effect of scale factor error and the $\alpha_{i i}$ soft iron terms denoted by $\gamma_{x}, \gamma_{y}$ and $\gamma_{z}$. The given or known inputs to the calibration algorithm are the measured magnetometer outputs, $\hat{h}_{x}^{b}, \hat{h}_{y}^{b}$, and $\hat{h}_{z}^{b}$, and the magnitude of Earth's magnetic field vector, $\|\vec{h}\|=h$, in the geographic area where the calibration is being performed.

\subsection{Least Squares Estimation}

We can fit an ellipsoid of revolution to measured magnetic field data by using a batch least squares estimator. The equations of the estimator can be obtained by linearizing Equation 18. The estimator will have as states perturbations of the ellipsoid parameters defined in Equations 18 through 21. Thus,

given an initial guess of the unknown parameters, the estimated perturbation are sequentially added to the initial guess and the procedure is repeated until convergence is achieved.

To linearize Equation 18, we note that the pertubation of $\|h\|$, written as $\delta h$, is given by [9]:

$$
\begin{aligned}
-\delta h & =\left(\frac{\hat{h}_{x}^{b}-b_{x}}{h \gamma_{x}^{2}}\right) \delta b_{x}+\left(\frac{\hat{h}_{x}^{b}-b_{x}}{\sqrt{h \gamma_{x}^{3}}}\right)^{2} \delta \gamma_{x} \\
& +\left(\frac{\hat{h}_{y}^{b}-b_{y}}{h \gamma_{y}^{2}}\right) \delta b_{y}+\left(\frac{\hat{h}_{y}^{b}-b_{y}}{\sqrt{h \gamma_{y}^{3}}}\right)^{2} \delta \gamma_{y} \\
& +\left(\frac{\hat{h}_{z}^{b}-b_{z}}{h \gamma_{z}^{2}}\right) \delta b_{z}+\left(\frac{\hat{h}_{z}^{b}-b_{z}}{\sqrt{h \gamma_{z}^{3}}}\right)^{2} \delta \gamma_{z} \\
& =\zeta_{x} \delta b_{x}+\eta_{x} \delta \gamma_{x}+\zeta_{y} \delta b_{y}+\eta_{y} \delta \gamma_{y}+\zeta_{z} \delta b_{z}+\eta_{z} \delta \gamma_{z},
\end{aligned}
$$


where $h=\|\vec{h}\|$. Note that the measured magnetometer outputs $\hat{h}_{x}^{b}, \hat{h}_{y}^{b}$, and $\hat{h}_{z}^{b}$ are functions of time even though, for the sake of clarity, we have dropped the explicit notation of time in Equation 23. That is, is should be noted that $\hat{h}_{y}^{b}$ is actually $\hat{h}_{y}^{b}\left(t=t_{k}\right)$ and corresponds to the $x$-magnetometer field measurement at time step $k$. The same is true for the $y$ and $z$ field measurements. Thus, given field measurements from $k$ time steps, Equation 23 can be written as:

$$
\left[\begin{array}{c}
\delta h_{1} \\
\delta h_{2} \\
\vdots \\
\delta h_{N}
\end{array}\right]=\left[\begin{array}{cccccc}
\zeta_{x_{1}} & \eta_{x_{1}} & \zeta_{y_{1}} & \eta_{y_{1}} & \zeta_{z_{1}} & \eta_{z_{1}} \\
\zeta_{x_{2}} & \eta_{x_{2}} & \zeta_{y_{2}} & \eta_{y_{2}} & \zeta_{z_{2}} & \eta_{z_{2}} \\
\zeta_{x_{3}} & \eta_{x_{3}} & \zeta_{y_{3}} & \eta_{y_{3}} & \zeta_{z_{3}} & \eta_{z_{3}} \\
\vdots & & \ddots & & & \vdots \\
\zeta_{x_{k-1}} & \eta_{x_{k-1}} & \zeta_{y_{k-1}} & \eta_{y_{k-1}} & \zeta_{z_{k-1}} & \eta_{z_{k-1}} \\
\zeta_{x_{k}} & \eta_{k_{N}} & \zeta_{k_{N}} & \eta_{k_{N}} & \zeta_{k_{N}} & \eta_{k_{N}}
\end{array}\right]\left[\begin{array}{c}
\delta b_{x} \\
\delta \gamma_{x} \\
\delta b_{y} \\
\delta \gamma_{y} \\
\delta b_{z} \\
\delta \gamma_{z}
\end{array}\right]
$$

Equation 24 is in the form $\delta \vec{h}=H \delta \vec{x}$ where $\delta \vec{x}$ is the vector of unknowns given by:

$$
\delta \vec{x}=\left[\begin{array}{llllll}
\delta b_{x} & \delta \gamma_{x} & \delta b_{y} & \delta \gamma_{y} & \delta b_{z} & \delta \gamma_{z}
\end{array}\right]^{T}
$$

The vector $\delta \vec{h}$ is the difference between the known magnetic field vector magnitude and its magnitude as computed from the magnetometer outputs. That is, $\delta h_{k}=h_{k}-\hat{h}_{k}$ where $h_{k}$ is computed from the IGRF model [8] and $\hat{h}_{k}$ is computed as:

$$
\hat{h}_{k}=\sqrt{\hat{h}_{x}^{b}+\hat{h}_{y}^{b}+\hat{h}_{y}^{b}}
$$

An estimate of the calibration parameters $\hat{b}_{x}, b_{y}, \hat{b}_{z}, \hat{\gamma}_{x}, \hat{\gamma}_{y}$, and $\hat{\gamma}_{z}$ is obtained by using the following iterative algorithm:

1. Select an initial guess for $\hat{b}_{x}, b_{y}, \hat{b}_{z}, \hat{\gamma}_{x}, \hat{\gamma}_{y}$, and $\hat{\gamma}_{z}$. The initial guess for $\gamma_{x}, \gamma_{y}$ and $\gamma_{z}$ must be non-zero.

2. Using the values of $\hat{b}_{x}, b_{y}, \hat{b}_{z}, \hat{\gamma}_{x}, \hat{\gamma}_{y}$, and $\hat{\gamma}_{z}$ form Equation 24 . 
3. Obtain a least squares estimate for $\delta \vec{x}$, denoted by $\delta \hat{\vec{x}}$, as follows:

$$
\delta \hat{\vec{x}}=\left(H^{T} H\right)^{-1} H^{T} \delta \vec{h}
$$

4. Use the estimate for $\delta \hat{\vec{x}}$ and update the unknown parameters as follows $("(+)$ " denotes parameter after update and "(-)" denotes parameter before update):

$$
\begin{aligned}
b_{x}(+) & =b_{x}(-)+\delta \hat{\vec{x}}(1) \\
\gamma_{x}(+) & =\gamma_{x}(-)+\delta \hat{\vec{x}}(2) \\
b_{y}(+) & =b_{y}(-)+\delta \hat{\vec{x}}(3) \\
\gamma_{y}(+) & =\gamma_{y}(-)+\delta \hat{\vec{x}}(4) \\
b_{z}(+) & =b_{z}(-)+\delta \hat{\vec{x}}(5) \\
\gamma_{z}(+) & =\gamma_{z}(-)+\delta \hat{\vec{x}}(6)
\end{aligned}
$$

5. Compute the covariance matrix $P$ (which is a measure of the quality of the calibration) by using

$$
P=\sigma_{w}^{2}\left(H^{T} H\right)^{-1}
$$

where $\sigma_{w}$ is the standard deviation of the magnetometer wide band noise.

6. Return to Step (2) and repeat until convergence is achieved. Convergence is achieved when the estimate of $b_{x}, b_{Y}, b_{z}, \gamma_{x}, \gamma_{x}$ and $\gamma_{x}$ do not change from one iteration to the next.

The estimated calibration parameters can now be used compute the corrected field measurements $h_{x}^{b}, h_{y}^{b}$ 
and $h_{z}^{b}$ from the measured magnetometer readings $\hat{h}_{x}^{b}, \hat{h}_{y}^{b}$ and $\hat{h}_{z}^{b}$ using the following relations:

$$
\begin{aligned}
h_{x}^{b} & =\frac{\hat{h}_{x}^{b}-\hat{b}_{x}}{\hat{\gamma}_{x}} \\
h_{y}^{b} & =\frac{\hat{h}_{y}^{b}-\hat{b}_{y}}{\hat{\gamma}_{y}} \\
h_{z}^{b} & =\frac{\hat{h}_{z}^{b}-\hat{b}_{z}}{\hat{\gamma}_{z}}
\end{aligned}
$$

As will be shown later in the paper, the stability of the least squares solution is sensitive to at least three factors. At this point, we will discuss one of these factor which is the quality of the initial conditions used to start the algorithm. The closer the initial guesses are to the actual value of the six parameters, the more stable the solution becomes. Since a judicious selection of initial conditions enhances the performance of the calibration, we will develop an algorithm that can be used to establish the initial conditions.

\subsection{Establishing Initial Conditions}

The algorithms used for establishing initial conditions, uses a non-linear, two-step estimator. This non-linear, two-step estimator is an adaptation of an estimator presented in [10] and breaks the parameter identification problem given by Equation 18 into two steps. In the first-step, a state vector called the "first-step state" is formed. The elements of this state vector are algebraic combinations of the elements of the "second-step state" vector. The elements of the second-step state vector, on the other hand, are hard iron biases and the scale factor-soft iron $\gamma$ terms. The estimation problem is linear in the firststep state and, therefore, retains the desirable properties of a linear system. Following estimation of the first-step states, elements of the second-step state vector are extracted through algebraic manipulation.

Derivation of the equations for the non-linear two-step estimator begin by expanding Equation 18 as 
follows:

$$
\begin{aligned}
h^{2}= & \frac{\left(\hat{h}_{x}^{b}\right)^{2}-2\left(\hat{h}_{x}^{b}\right)\left(b_{x}\right)+\left(b_{x}\right)^{2}}{\gamma_{x}^{2}} \\
& +\frac{\left(\hat{h}_{y}^{b}\right)^{2}-2\left(\hat{h}_{y}^{b}\right)\left(b_{y}\right)+\left(b_{y}\right)^{2}}{\gamma_{y}^{2}} \\
& +\frac{\left(\hat{h}_{z}^{b}\right)^{2}-2\left(\hat{h}_{z}^{b}\right)\left(b_{z}\right)+\left(b_{z}\right)^{2}}{\gamma_{z}^{2}} .
\end{aligned}
$$

Note that the field measurements are a function of time. Given $k$ field measurements, we can construct $k$ separate equations like Equation 38. Rearranging $k$ Equation 38 like expressions into a matrix equation of the standard $\vec{z}=H \vec{x}+\vec{v}$ form leads to:

$$
-\left[\begin{array}{c}
\left(\hat{h}_{x}^{b}\left(t_{1}\right)\right)^{2} \\
\left(\hat{h}_{x}^{b}\left(t_{2}\right)\right)^{2} \\
\left(\hat{h}_{x}^{b}\left(t_{3}\right)\right)^{2} \\
\vdots \\
\left(\hat{h}_{x}^{b}\left(t_{k-1}\right)\right)^{2} \\
\left(\hat{h}_{x}^{b}\left(t_{k}\right)\right)^{2}
\end{array}\right]=\left[\begin{array}{ll}
H_{11} & H_{12}
\end{array}\right]\left[\begin{array}{c}
b_{x} \\
\mu_{2}\left(b_{y}\right) \\
\mu_{3}\left(b_{z}\right) \\
\mu_{1} \\
\mu_{3} \\
\mu_{4}
\end{array}\right]+\vec{v} .
$$

The vector $\vec{v}$ represents the measurement noise. The measurement matrix $H$ consists of two $k \times 3$ sub-matrices. The first of these two sub-matrices, $H_{11}$, is defined as:

$$
H_{11}=\left[\begin{array}{ccc}
-2 \hat{h}_{x}^{b}\left(t_{1}\right) & -2 \hat{h}_{y}^{b}\left(t_{1}\right) & -2 \hat{h}_{z}^{b}\left(t_{1}\right) \\
-2 \hat{h}_{x}^{b}\left(t_{2}\right) & -2 \hat{h}_{y}^{b}\left(t_{2}\right) & -2 \hat{h}_{z}^{b}\left(t_{2}\right) \\
-2 \hat{h}_{x}^{b}\left(t_{3}\right) & -2 \hat{h}_{y}^{b}\left(t_{3}\right) & -2 \hat{h}_{z}^{b}\left(t_{3}\right) \\
\vdots & \vdots & \vdots \\
-2 \hat{h}_{x}^{b}\left(t_{k-1}\right) & -2 \hat{h}_{y}^{b}\left(t_{k-1}\right) & -2 \hat{h}_{z}^{b}\left(t_{k-1}\right) \\
-2 \hat{h}_{x}^{b}\left(t_{k}\right) & -2 \hat{h}_{y}^{b}\left(t_{k}\right) & -2 \hat{h}_{z}^{b}\left(t_{k}\right)
\end{array}\right]
$$


The second sub-matrix $H_{12}$ is given by:

$$
H_{12}=\left[\begin{array}{ccc}
\left(\hat{h}_{y}^{b}\left(t_{1}\right)\right)^{2} & \left(\hat{h}_{z}^{b}\left(t_{1}\right)\right)^{2} & 1 \\
\left(\hat{h}_{y}^{b}\left(t_{2}\right)\right)^{2} & \left(\hat{h}_{z}^{b}\left(t_{2}\right)\right)^{2} & 1 \\
\left(\hat{h}_{y}^{b}\left(t_{3}\right)\right)^{2} & \left(\hat{h}_{z}^{b}\left(t_{3}\right)\right)^{2} & 1 \\
\vdots & \vdots & \vdots \\
\left(\hat{h}_{y}^{b}\left(t_{k-1}\right)\right)^{2} & \left(\hat{h}_{z}^{b}\left(t_{k-1}\right)\right)^{2} & 1 \\
\left(\hat{h}_{y}^{b}\left(t_{k}\right)\right)^{2} & \left(\hat{h}_{z}^{b}\left(t_{k}\right)\right)^{2} & 1
\end{array}\right]
$$

The vector on the right side of Equation 39, denoted as $\vec{x}$, is the first-step state vector and consists of the variables $\mu_{1}$ through $\mu_{4}$ which are defined as follows:

$$
\begin{aligned}
\mu_{1} & =h^{2} \gamma_{x}^{2} \\
\mu_{2} & =\frac{\gamma_{x}^{2}}{\gamma_{y}^{2}} \\
\mu_{3} & =\frac{\gamma_{x}^{2}}{\gamma_{z}^{2}} \\
\mu_{4} & =\left(b_{x}\right)^{2}+\mu_{2}\left(b_{y}\right)^{2}+\mu_{3}\left(b_{z}\right)^{2}-\mu_{1} .
\end{aligned}
$$

An estimate for $\vec{x}$, denoted as $\hat{\vec{x}}$, is obtained by:

$$
\hat{\vec{x}}=\left(H^{T} H\right)^{-1} H^{T} \vec{y}
$$

Once the first-step state vector is estimated, $\hat{b}_{x}, b_{y}, \hat{b}_{z}, \hat{\gamma}_{x}, \hat{\gamma}_{y}$, and $\hat{\gamma}_{z}$ are extracted from $\hat{\vec{x}}$ by the following 
inverse relations:

$$
\begin{aligned}
& \hat{b}_{x}=\hat{\vec{x}}(1) \\
& \hat{b}_{y}=\frac{\hat{\vec{x}}(2)}{\hat{\vec{x}}(4)} \\
& \hat{b}_{z}=\frac{\hat{\vec{x}}(3)}{\hat{\vec{x}}(5)} \\
& \hat{\gamma_{x}}=\sqrt{\frac{\mu_{1}}{h^{2}}} \\
& \hat{\gamma}_{y}=\sqrt{\frac{\mu_{1}}{\mu_{2} h^{2}}} \\
& \hat{\gamma}_{z}=\sqrt{\frac{\mu_{1}}{\mu_{3} h^{2}}}
\end{aligned}
$$

It was found that Equations 39 through 52 provide a very good estimate of the calibration parameters. It is conceivable, therefore, that this initialization algorithm alone can be used as a "snap-shot" solution in lieu of the iterative least squares solution developed earlier. This was avoided, however, because the two-step formulation as discussed here does not provide for an easy way to compute the posterior covariance matrix $P$ which will be used as a metric for the quality of the calibration. This is because the measurement noise vector $\vec{v}$ is the result of squaring the outputs of the magnetometers (i.e. vector on the right side of Equation 39) and thus is neither zero mean nor Gaussian distributed. While novel, nonlinear estimation techniques which combine the unscented transformation [11] with Kalman or Particle Filtering $[12,13]$ can be used to directly estimate the posterior covariance matrix, for the work reported in this paper, we will use the linearized estimate of $P$ given by Equation 34. As will be shown in the next section, this estimate of $P$ generally tends to over bound the actual estimation errors at a $1-\sigma$ level.

\section{SIMULATION STUDIES}

A series of simulation studies was performed to assess the performance of the magnetometer calibration algorithms. The results show that the iterative batch least squares estimator is primarily sensitive to three factors. First, the algorithm is sensitive to initial values; unless the hard iron biases and $\gamma$ factors 
close to their actual values, the algorithm can diverge. Second, the algorithm is sensitive to sampling and sensor noise. The amount of noise that can be tolerated is, however, a function of the third factor which is the shape of the measurement locus. Each of these sensitivity factors will be discussed next.

It will be recalled that the iterative batch least squares estimator requires an initial guess of hard iron biases $(b)$ and scale-factor/soft iron error terms $(\gamma)$. The first set of simulation studies evaluated the performance of the algorithm where the initial conditions were chosen randomly without using the two-step non-linear estimator. The initial guesses for the hard iron biases were picked from a normal distribution with a mean equal to the actual biases and a standard deviation of 0.5 Gauss. Similarly, the initial guesses for the scale-factor/soft iron error term, $\gamma$, were picked from a normal distribution centered at the actual value of $\gamma$ with a standard deviation 0.5 .

Two values of measurement noise standard deviation, $\sigma_{w}$, were evaluated. One of the values considered was 5 milli-Gauss and is based on the data shown in Figure 2. The data shown in Figure 2, however, was collected on a system that used a digital pre-filter to process that field measurements before they were recorded. In the absence of such a filter, the value of the wide band noise can be as high at 10 milli-Gauss. As such, the second value of $\sigma_{w}$ considered was 10 mili-Gauss.

Figure 5 is a schematic that illustrates the metric used for quantifying the measurement locus geometry. If during the calibration procedure the magnetometer assembly is rotated through space such that the entire Euler angle space is spanned, the locus of magnetometer measurements obtained would be as shown in Figure 5(a). As can be seen from the experimental data shown in Figure 6, this is not always possible. Figure 6 shows that only a small portion of the ellipsoid is present. Thus, for the simulation studies we will also assume that only a small portion of the ellipsoid is present (Figure 5(b)). The central angle spanned by the strip of the ellipsoid, $\Phi$, is used to characterize the geometry of the measurement locus (Figure 5(c)).

Table 1 shows the four cases simulated study to quantify estimation accuracy as a function of the three sensitivity factors discussed above. The results of these trade-off studies are shown in Figures 7 through 13. Figures 7 and 8 show the performance of the iterative batch least squares estimator in the presence of a 5 milli-Gauss wide-band noise and when $10^{\circ}$ and $20^{\circ}$ strips of measurement locus are 


\begin{tabular}{|c|c|c|c|c|c|}
\hline $\begin{array}{c}\text { Case \& Strip } \\
\text { Size }\end{array}$ & \multicolumn{2}{|c|}{$\begin{array}{c}\text { Hard Iron } \\
\text { Bias } \\
\end{array}$} & \multicolumn{2}{|c|}{$\begin{array}{c}\text { Scale Factor \& } \\
\text { Soft Iron }\end{array}$} & $\begin{array}{c}\text { Wide-Band } \\
\text { Noise }\end{array}$ \\
\hline \multirow{3}{*}{$\mathrm{I}, 10^{\circ}$} & $b_{x}$ & 1 Gauss & $\gamma_{x}$ & 4 & \multirow{3}{*}{5 milli-Gauss } \\
\hline & $b_{y}$ & 2 Gauss & $\gamma_{y}$ & 3 & \\
\hline & $b_{z}$ & -3 Gauss & $\gamma_{z}$ & 2 & \\
\hline \multirow{3}{*}{ II, $20^{\circ}$} & $b_{x}$ & 1 Gauss & $\gamma_{x}$ & 4 & \multirow{3}{*}{5 milli-Gauss } \\
\hline & $b_{y}$ & 2 Gauss & $\gamma_{y}$ & 3 & \\
\hline & $b_{z}$ & -3 Gauss & $\gamma_{z}$ & 2 & \\
\hline \multirow{3}{*}{ III, $10^{\circ}$} & $b_{x}$ & 1 Gauss & $\gamma_{x}$ & 4 & \multirow{3}{*}{10 milli-Gauss } \\
\hline & $b_{y}$ & 2 Gauss & $\gamma_{y}$ & 3 & \\
\hline & $b_{z}$ & -3 Gauss & $\gamma_{z}$ & 2 & \\
\hline \multirow{3}{*}{$\mathrm{IV}, 20^{\circ}$} & $b_{x}$ & 1 Gauss & $\gamma_{x}$ & 4 & \multirow{3}{*}{10 milli-Gauss } \\
\hline & $b_{y}$ & 2 Gauss & $\gamma_{y}$ & 3 & \\
\hline & $b_{z}$ & -3 Gauss & $\gamma_{z}$ & 2 & \\
\hline
\end{tabular}

Table 1: Parameters for Magnetometer Calibration Simulations.

available. The algorithm is seen to converge in both cases. When the measurement noise is increased to 10 milli-Gauss, the results shown in Figures 9 and 10 are obtained. Figure 11 shows the results from another run with a $10^{\circ}$ strip and 10 mili-Gauss of wide band noise. In this case, it is seen that the algorithm diverges when only a $10^{\circ}$ strip of the measurement locus is available. Actually, of fact the algorithm diverges just about as frequently as it converges with the available measurement locus is small and the wide band noise is large. The algorithm also diverged when $\Phi=20^{\circ}$ but not as often as it did when $\Phi=10^{\circ}$. The divergence is primarily due to the initial conditions assigned to hard iron biases and $\gamma$ being too far away from the actual values. This becomes apparent when we note that in Figures 9, 10 and 11 the initial conditions are not the same because, as noted earlier, the initial conditions were varied randomly from one try to the next. Observe, for example, that the initial conditions assigned to $b_{x}$ and $b_{y}$ for the run shown in Figure 9 are closer to the actual values than assigned for the run shown in Figure 11.

The difference in initial conditions, however, is not the only cause of the divergence because these plots show the results for just one simulation run out of many. The algorithm diverged repeatedly when only a $10^{\circ}$ strip of the locus was used while, it converged more often when a $20^{\circ}$ strip of the measurement locus was available. As noted earlier, the measurement noise on low cost magnetometers can be as high 
as the 10 milli-Gauss figure used in some of these simulations. Therefore, it is concluded that for relatively low cost magnetometers with relatively large magnitude output noise, this algorithm is not suitable unless a large portion of the ellipsoid is available.

This sensitivity to measurement locus geometry has a very important practical implication. When discussing the methods for calibrating a two-magnetometer system, it was noted that the parameter estimation problem is one where the best circle (in the least squares sense) is fit to the noisy magnetometer measurement data. A simple $360^{\circ}$ turn on a level surface yielded the required measurement locus. In extending this method to the three-dimensional case, a tacit assumption was that the entire sphere would be available for the parameter estimation problem. Unfortunately this is not always the case because getting a complete sphere requires spanning the entire Euler angle space. Thus, unless the magnetometer triad is installed in an aerobatic airplane, spanning the entire Euler angle space is not possible. So the three dimensional calibration algorithms must be able to work with data that comprises only a portion of the entire sphere. Actual data collected from a flight test is shown in Figure 6. It is clear from this figure that an entire ellipsoid can not be obtained in a non-aerobatic aircraft.

The simulation results for the cases where the non-linear, two-Step estimator is used to initialize the iterative batch least squares estimator are shown in Figures 12 and 13. These figures are histograms for the bias and $\gamma$ estimation errors (or residuals) for 10,000 Monte Carlo simulation runs. For each run, simulated magnetometer outputs were corrupted with bias and scale factor errors as well as wide-band noise. The bias and scale factor errors were held constant for all 10,000 simulation runs and had the values given in Table 1. The wide-band noise, however, was varied for each run. It was a random sequence with a standard deviation of 5 or 10 milli-Gauss.

Figure 12 shows the bias estimation residuals. In none of the 10,000 cases did the solution diverge. For the $x$ and $y$ axes hard iron biases, the estimation errors are seen to be less than \pm 0.5 milli-Gauss. In comparison to the $x$ and $y$ axes, the $z$ axis estimation errors are slightly larger. However, this error is smaller when the locus of magnetometer measurements is larger. A similar trend is seen in Figure 13 which shows the scale factor estimation errors. The fact that the estimation errors for both the $z$ axis hard iron biases and scale factor errors are larger in comparison to the $x$ and $y$ axes errors is not surprising 


\begin{tabular}{|c||c|c||c|c||}
\hline $\begin{array}{c}\text { Simulation } \\
\text { Case }\end{array}$ & \multicolumn{2}{c||}{$\begin{array}{c}\text { Bias Estimation } \\
\text { Error }\left(\times 10^{-3} \text { Gauss }\right)\end{array}$} & \multicolumn{2}{c|}{$\begin{array}{c}\gamma \text { Estimation } \\
\text { Error (no units) }\end{array}$} \\
\hline \hline \multirow{3}{*}{ I } & $\delta b_{x}$ & 1.73 & $\delta \gamma_{x}$ & 0.00549 \\
\cline { 2 - 5 } & $\delta b_{y}$ & 1.30 & $\delta \gamma_{y}$ & 0.00410 \\
\cline { 2 - 5 } & $\delta b_{z}$ & 9.00 & $\delta \gamma_{z}$ & 0.361 \\
\hline \hline \multirow{2}{*}{ II } & $\delta b_{x}$ & 1.34 & $\delta \gamma_{x}$ & 0.00402 \\
\cline { 2 - 5 } & $\delta b_{y}$ & 1.01 & $\delta \gamma_{y}$ & 0.00304 \\
\cline { 2 - 5 } & $\delta b_{z}$ & 3.90 & $\delta \gamma_{z}$ & 0.0756 \\
\hline \hline \multirow{3}{*}{ III } & $\delta b_{x}$ & 3.46 & $\delta \gamma_{x}$ & 0.0108 \\
\cline { 2 - 5 } & $\delta b_{y}$ & 2.60 & $\delta \gamma_{y}$ & 0.00809 \\
\cline { 2 - 5 } & $\delta b_{z}$ & 17.0 & $\delta \gamma_{z}$ & 0.631 \\
\hline \hline \multirow{3}{*}{ IV } & $\delta b_{x}$ & 2.70 & $\delta \gamma_{x}$ & 0.00805 \\
\cline { 2 - 5 } & $\delta b_{y}$ & 2.03 & $\delta \gamma_{y}$ & 0.00606 \\
\cline { 2 - 5 } & $\delta b_{z}$ & 7.55 & $\delta \gamma_{z}$ & 0.143 \\
\hline
\end{tabular}

Table 2: State Estimation Errors $(1-\sigma)$.

because even in the $20^{\circ}$ locus case the data spans only a small amount of space in the $z$ direction.

The $1-\sigma$ standard deviation of the hard iron bias and $\gamma$ estimation errors for one representative run are summarized in Table 2. The $1-\sigma$ estimation errors are nothing more than the square root of the diagonal elements of the covariance matrix $P$. As noted earlier and is confirmed by observing Figure 12, Figure 13, and the data in Table 2, the estimated covariance over bounds the actual errors. Thus, $P$ can be used as conservative metric for the quality of the calibration.

In conclusion, initializing the iterative batch least squares estimator using the Non-Linear, two-Step Estimator is seen to provide superior performance. More specifically, the algorithm does not diverge even in the case when the wide-band noise on the magnetometer measurements is large. Thus, it requires a smaller portion of the measurement locus than the case where the iterative least squares algorithm is used alone.

\section{EXPERIMENTAL RESULTS}

As a final verification, a triad of low cost magnetometers was calibrated using the algorithms developed in this paper. The data was collected from an experimental set up where a set of low cost mag- 
netometers were strapped to a long wooden boom as shown in Figure 14. The wooden boom was used in order to isolate the magnetometers from magnetic field generating electronics in the data collecting computer and associated hardware. To verify the quality of the calibration, the post-calibration heading solution was compared with the heading solution from an expensive navigation grade INS (Honeywell YG1851 IRU). The INS and the experimental set up are shown in Figure 14.

Figure 15 shows a histogram of the residual in the magnetic field domain after the calibration is complete. These residuals were computed by resolving the known magnetic field vector in the area where this calibration took place (i.e., the San Francisco Bay Area) and resolving it into the axes of the magnetometer triad using the precise INS attitude information. The largest residual, which is on the x-axis magnetometer, has a mean of -0.007 Gauss and a standard deviation of 0.004 Gauss.

Figure 16 shows a one-minute trace comparing the heading solution computed using the magnetometers with the heading solution generated by the INS. The heading residuals for this one-minute trace are less than $3^{\circ}$ RMS. Figure 17 is a histogram of the heading errors for the entire experiment. It is seen that the heading error has a standard deviation of $3.6^{\circ}$ and a mean of $1.2^{\circ}$. The largest heading error observed was $18^{\circ}$ and was the result of the wooden boom flexing relative the the INS (i.e., the truth reference) during the data collection maneuvers. Once the mean is removed, the remaining heading error is, to a large extent, in the form of wide-band noise which can be easily filtered using a a low-pass filter.

\section{CONCLUSIONS}

An algorithm for calibrating strapdown magnetometers used in heading determination systems was developed. Unlike the classic method of compass swinging which computes a series of heading correction parameters, this algorithm estimates magnetometer output errors directly and, thus, is not limited to heading determination systems.

The calibration algorithm uses an estimator where the states are the hard iron biases, soft iron biases, and scale factor errors. The estimator is a linearized, iterative batch least squares estimator. The initial conditions for the estimator are established using a non-linear, two-step estimator. When initialized 
thusly, monte carlo simulations show the estimation procedure to be very robust.

As presented in this paper, the calibration algorithm is limited to estimation of the hard iron biases and combined scale factor and some soft iron effects. However, it should be possible to extend the applicability of this method to all magnetometer errors including misalignment and all soft iron errors.

\section{ACKNOWLEDGEMENTS}

The authors wish to acknowledge the FAA Satellite Navigation Product Team and The Office of Technology and Licensing at Stanford University for sponsoring the research reported in this paper.

\section{A APPENDIX: COMPASS SWINGING EQUATIONS}

In this appendix, we derive Equations 6 and 7 which are used in the compass swinging algorithm. In what follows, it will be assumed that the pair of magnetometers are level and there are no misalignment errors. The effect of misalignment errors will be considered later. A level and error-free pair of magnetometers measures the strength of the horizontal component of the local Earth magnetic field vector, $\vec{h}_{h}$. The measurement made by each of the magnetometers will be:

$$
\begin{aligned}
h_{x}^{b} & =h_{h} \cos (\psi) \\
h_{y}^{b} & =-h_{h} \sin (\psi) .
\end{aligned}
$$

where $h_{h}=\left\|\vec{h}_{h}\right\|$ Since the magnetometer assembly is assumed to be level, the body coordinate system is the same as the wander-azimuth coordinate system and $h_{x}^{b}$ and $h_{y}^{b}$ can be used in lieu of $h_{x}^{w}$ and $h_{y}^{w}$ in Equation 1.

If measurement errors are present, the output from the magnetometers in the body frame will not be 
equal to $h_{x}^{b}$ and $h_{y}^{b}$ given by Equations 53 and 54. Instead, the output of the x-magnetometer will be $\hat{h}_{x}^{b}$ :

$$
\begin{aligned}
\hat{h}_{x}^{b} & =h_{x}^{b}+\delta h_{x_{0}}+\alpha_{x x} h_{x}^{b}+\alpha_{x y} h_{y}^{b} \\
& =h_{x}^{b}+\delta h_{x_{0}}+\alpha_{x x} h_{H} \cos \psi-\alpha_{x y} h_{h} \sin \psi,
\end{aligned}
$$

Similarly, the y-magnetometer output will be $\hat{h}_{y}^{b}$ :

$$
\begin{aligned}
\hat{h}_{y}^{b} & =h_{y}^{b}+\delta h_{y_{0}}+\alpha_{y x} h_{x}^{b}+\alpha_{y y} h_{y}^{b} \\
& =h_{y}^{b}+\delta h_{y_{0}}+\alpha_{y x} h_{H} \cos \psi-\alpha_{y y} B_{H} \sin \psi
\end{aligned}
$$

The terms $\delta h_{x_{0}}$ and $\delta h_{y_{0}}$ represent the hard iron biases while the remaining terms account for errors due to soft iron. Our objective is to evaluate heading errors as function of magnetometer measurement errors. An expression relating heading errors to magnetometer measurement errors can be arrived at by taking a perturbation of Equation 1. This leads to:

$$
\begin{aligned}
\delta \psi & =\left(\frac{\partial \psi}{\partial h_{y}}\right) \delta h_{y}+\left(\frac{\partial \psi}{\partial h_{x}}\right) \delta h_{x} \\
& =-\frac{1}{h_{h}}\left(\delta h_{x} \sin \psi+\delta h_{y} \cos \psi\right)
\end{aligned}
$$

The perturbation quantities $\delta h_{x}$ and $\delta h_{y}$ represent magnetometer measurement errors and are given by:

$$
\begin{aligned}
& \delta h_{x}=\hat{h}_{x}^{b}-h_{x}^{b} \\
& \delta h_{y}=\hat{h}_{y}^{b}-h_{y}^{b}
\end{aligned}
$$

Substituting these values into Equation 57 and rearranging leads to the following equation for heading 
error:

$$
\begin{aligned}
\delta \psi= & \left(\frac{\alpha_{x y}-\alpha_{y y}}{2}\right)-\frac{\delta h_{x_{0}}}{h_{h}} \sin (\psi)-\frac{\delta h_{y_{0}}}{h_{h}} \cos (\psi) \\
& +\left(\frac{\alpha_{x x}-\alpha_{y x}}{2}\right) \sin (2 \psi)+\left(\frac{\alpha_{y y}-\alpha_{x y}}{2}\right) \cos (2 \psi) .
\end{aligned}
$$

This reduces to Equation 6 when the following substitutions are made:

$$
\begin{aligned}
& A=\left(\frac{\alpha_{x y}-\alpha_{y y}}{2}\right) \\
& B=\frac{\delta h_{x_{0}}}{h_{h}} \\
& C=\frac{\delta h_{y_{0}}}{h_{h}} \\
& D=\left(\frac{\alpha_{x x}-\alpha_{y x}}{2}\right) \\
& E=\left(\frac{\alpha_{y y}-\alpha_{x y}}{2}\right)
\end{aligned}
$$

Up to this point, misalignments have been ignored. Misalignment errors can be classified into two categories. The first category is the case of pitch and roll misalignment. Pitch and roll misalignments are installation errors that result in the magnetometers not being level when the vehicle is level. The two dimensional swinging algorithm cannot deal with with pitch and roll misalignments because the errors introduced by such misalignments are time varying. The second category is a yaw misalignment. This is the case where installation errors result in the magnetometer assembly being installed with an azimuth bias. Mathematically, a constant azimuth bias, $\delta \psi_{0}$, due to installation errors modifies Equation 60 in the following manner:

$$
\delta \psi=A+B \sin \left(\psi+\delta \psi_{0}\right)+C \cos \left(\psi+\delta \psi_{0}\right)+D \sin \left(2 \psi+2 \delta \psi_{0}\right)+E \cos \left(2 \psi+2 \delta \psi_{0}\right)
$$


When this equation is expanded using trigonometric identities and rearranged, one gets

$$
\delta \psi=\bar{A}+\bar{B} \sin (\psi)+\bar{C} \cos (\psi)+\bar{D} \sin (2 \psi)+\bar{E} \cos (2 \psi),
$$

which is identical to Equation 6 except that the coefficients are now modified. Thus, swinging can deal with yaw misalignments. This also implies that a compass rose is not really required when using a swinging algorithm. All that is required is to swing the magnetometer assembly through equally spaced headings around the compass rose followed by one final known heading. In this instance, the offset term, $\delta \psi_{0}$, will be the sum of the installation error and the constant heading error introduced by the fact that a compass rose was not used. Thus, the final known heading is used to separate the two individual components of $\delta \psi_{0}$. 


\section{References}

[1] Nathaniel Bowditch. The American Practical Navigator. Defense Mapping Agency, Hydrographic/Topographic Center, Bethesda, Maryland, USA, 1995.

[2] LITEF Corporation. LCR-92 Attitude Heading Reference System. LITEF Corporatoin, Freiberg, Germany, 2001.

[3] D. Gebre-Egziabher, G. H. Elkaim, J. D. Powell, and B. W. Parkinson. A Gyro-Free, Quaternion Based Attitude Determination System Suitable for Implementation Using Low-Cost Sensors. In Proceedings of the IEEE Position Location and Navigation Symposium, PLANS 2000, pages 185 192. IEEE, 2000.

[4] Demoz Gebre-Egziabher. Design and Performance Analysis of a Low-Cost Aided-Dead Reckoning Navigation System. PhD thesis, Department of Aeronautics and Astronautics, Stanford University, Stanford, California 94305, December 2001.

[5] Gabriel H. Elkaim. System Identification for Precision Control of a GPS-Autonomous Catamaran. PhD thesis, Stanford University, Stanford, California 94305, August 2001.

[6] Michael J. Caruso. Application of Magnetic Sensors for Low Cost Compass Systems. In Proceedings of the IEEE Position Location and Navigation Symposium, PLANS 2000, pages 177 - 184. IEEE, 2000.

[7] Myron Kayton and Walter R. Fried. Avionics Navigation Systems. John Wiley and Sons, Inc, New York, New York, $2^{\text {nd }}$ edition, 1997.

[8] C.E. Barton. Revision of International Geomagnetic Reference Field Release. EOS Transactions, 77(16), April 1996.

[9] Wilfred Kaplan. Advanced Calculus, pages 86 - 88. Addison-Wesley, Reading, Massachusetts, USA, 1952. 
[10] Gordon Thomas Haupt. Development and Experimental Verification of A Nonlinear Data Reduction Algorithm for Gravity Probe B Relativity Mission. PhD thesis, Stanford University, Stanford, California 94305, March 1996.

[11] S. J. Julier. The Scaled Unscented Transformation. In Proceedings of the American Control Conference. ACC, 2002.

[12] S. J. Julier and J. K. Uhlmann. A New Extenstion of the Kalman Filter to Nonlinear Systems. In Proceedings of AeroSense: The $11^{\text {th }}$ International Symposium on Aerospace/Defence Sening, Simulation and Controls, Orlando, Florida. AerSense, 1997.

[13] R. van der Merwe, A. Doucet, J. F. G. de Freitas, and E. Wan. The Unscented Particle Filter. Technical Report CUED/F-INFENG/TR 380, Cambridge University, Engineering Department, Cambridge, UK, August 2000. 


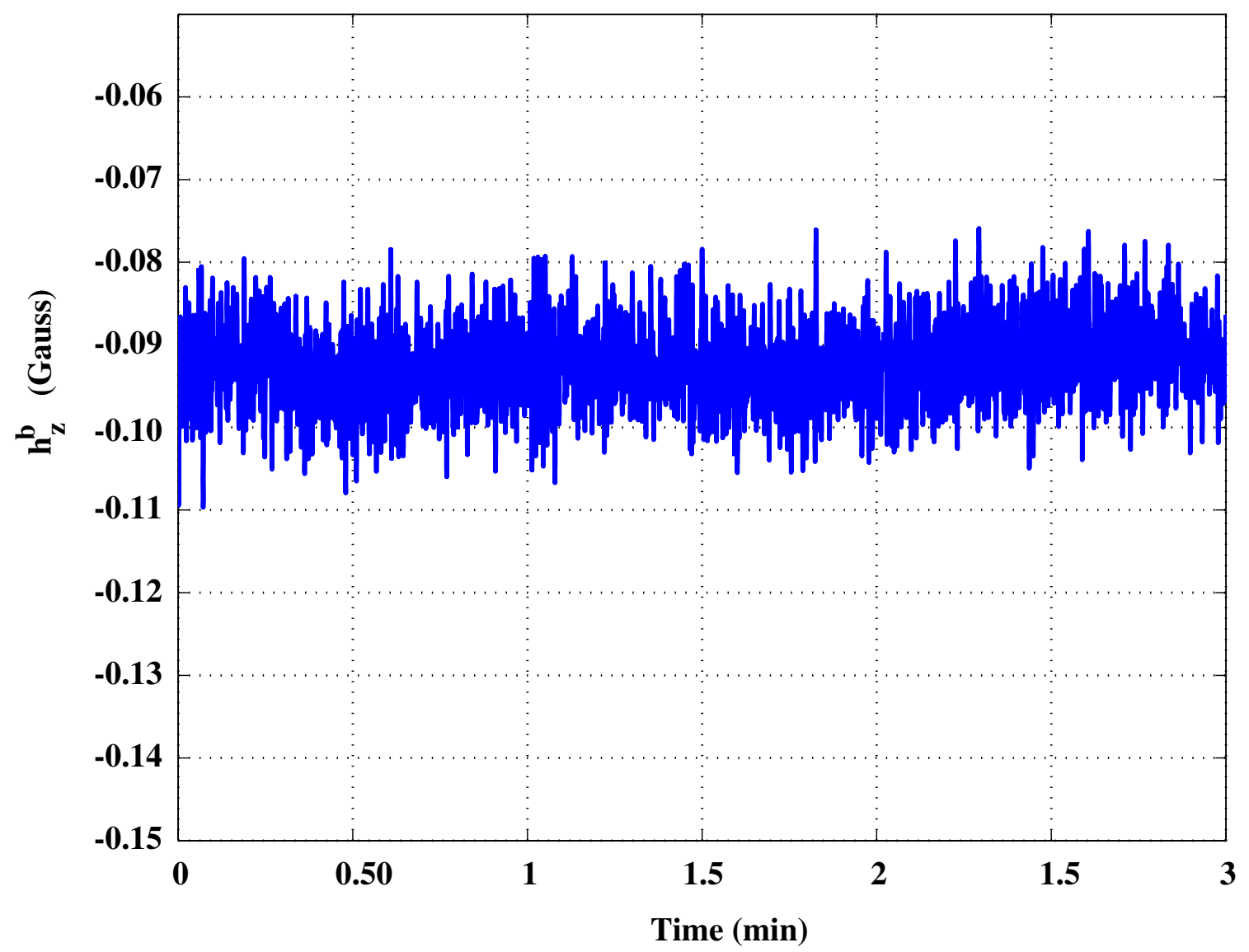

Figure 1: Magnetometer Null-Shift Time History (Output from a Honeywell HMC2003 Magnetometer Triad). 


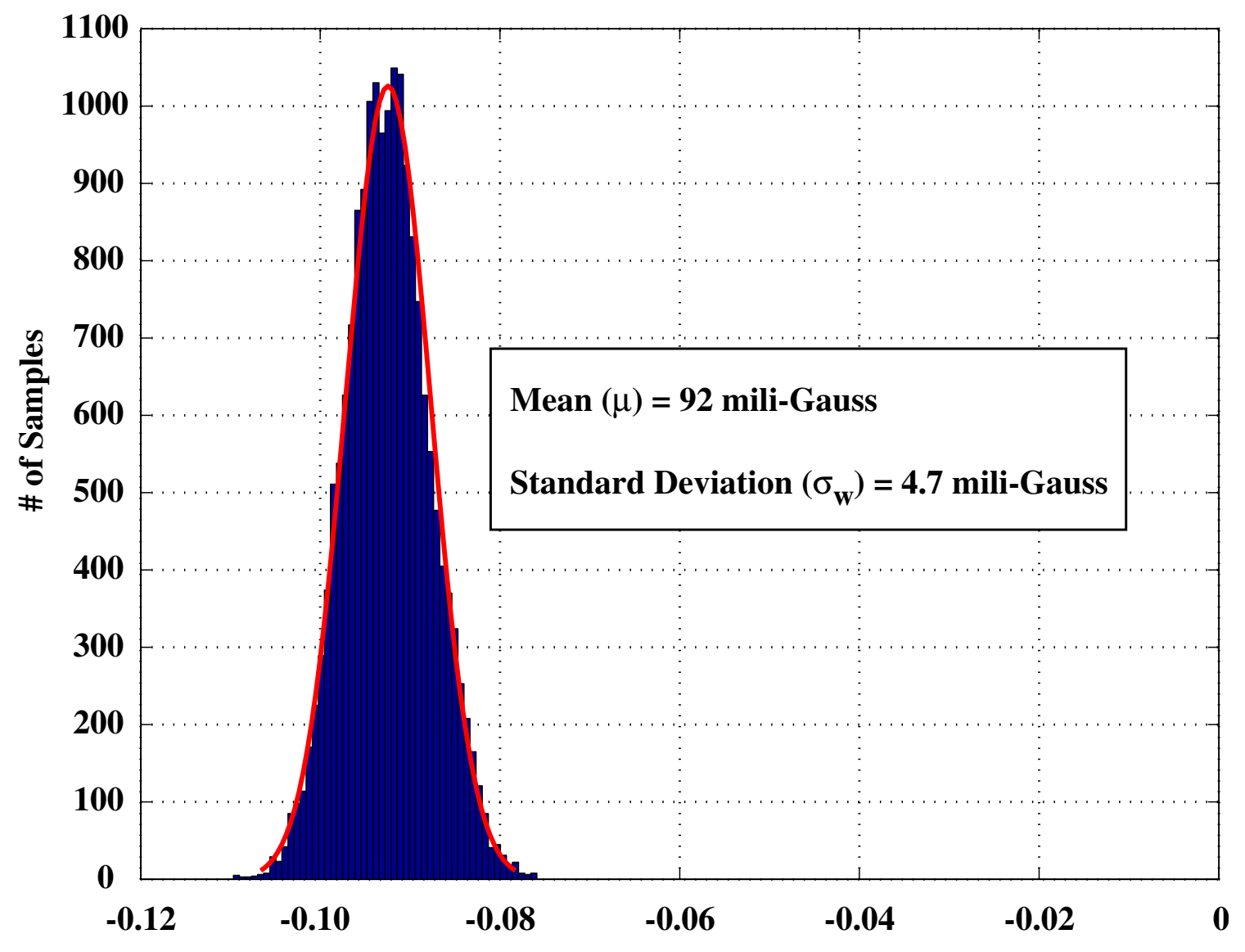

Figure 2: Histogram of the Magnetometer Output Error shown in Figure 1.

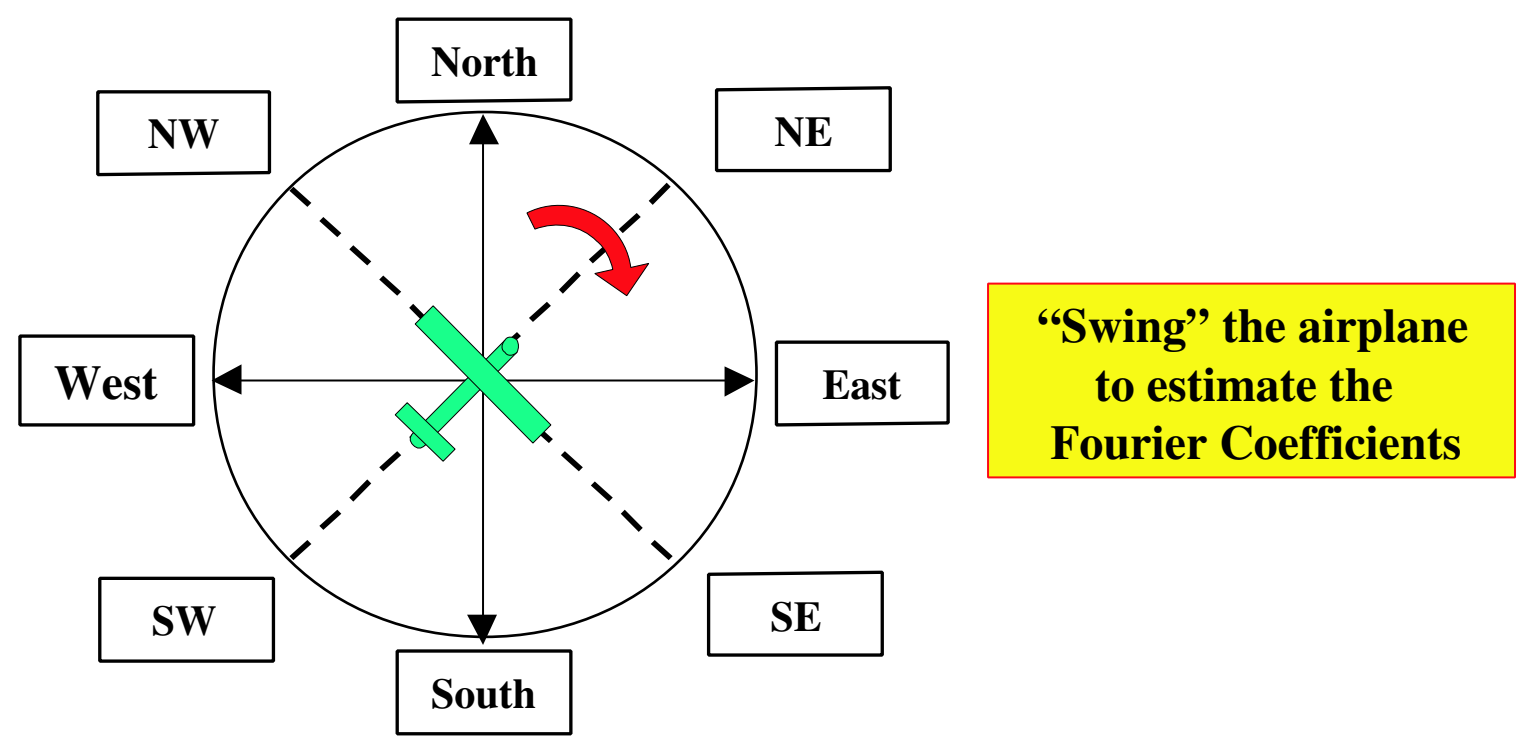

Figure 3: Graphical Description of Swinging. 


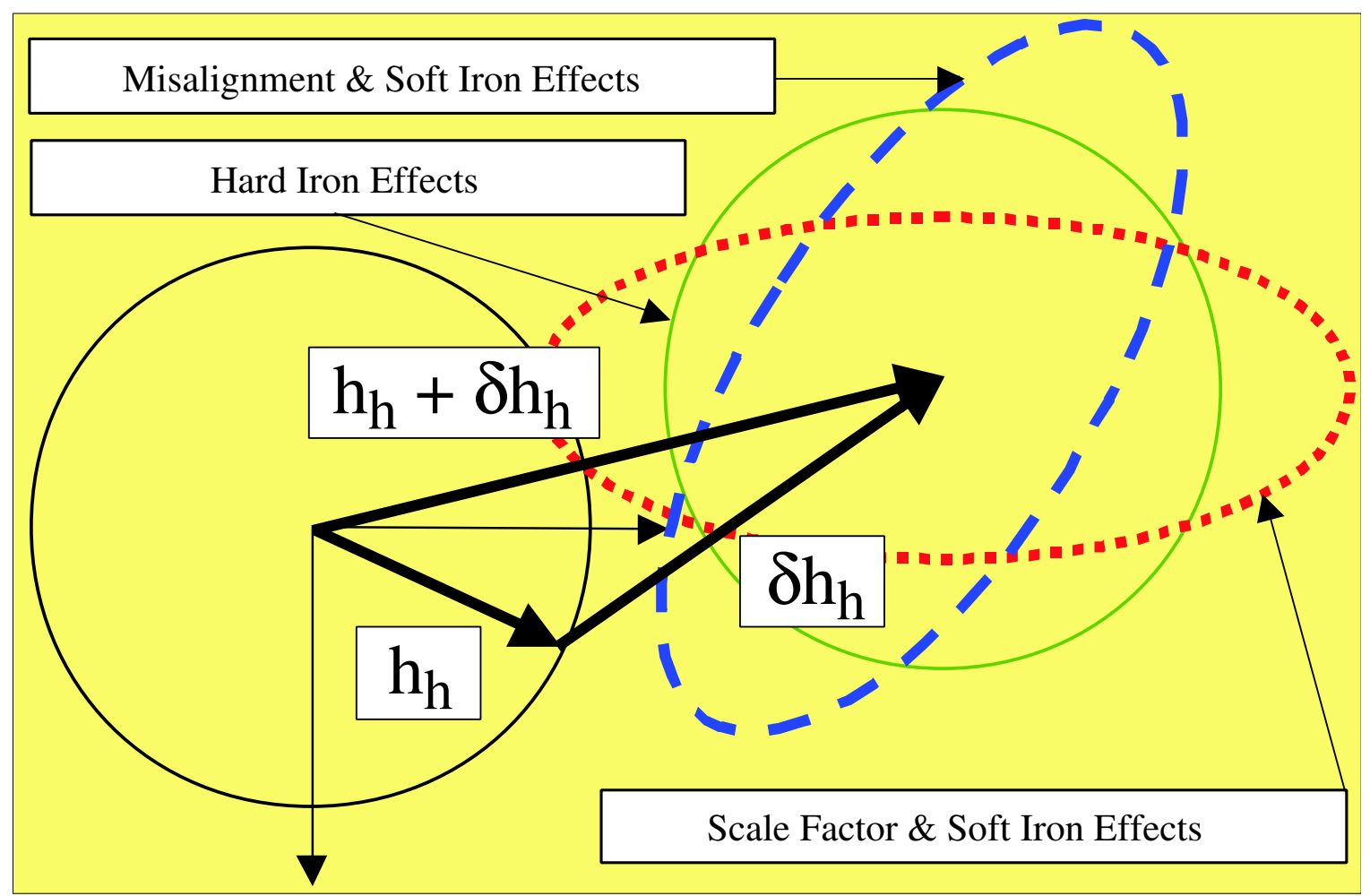

Figure 4: Effect of Errors on Magnetic Field Measurement Locus in 2-D. 


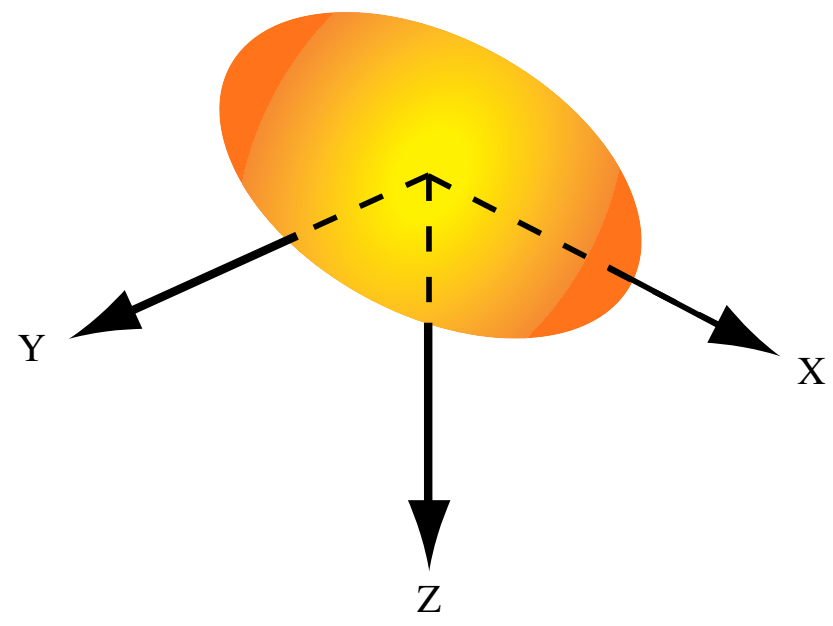

(a)

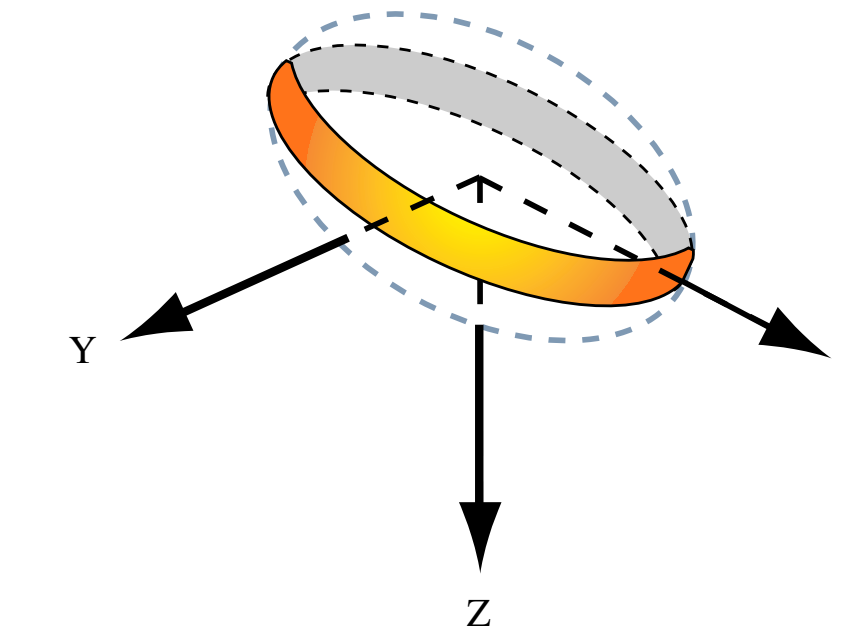

(b)

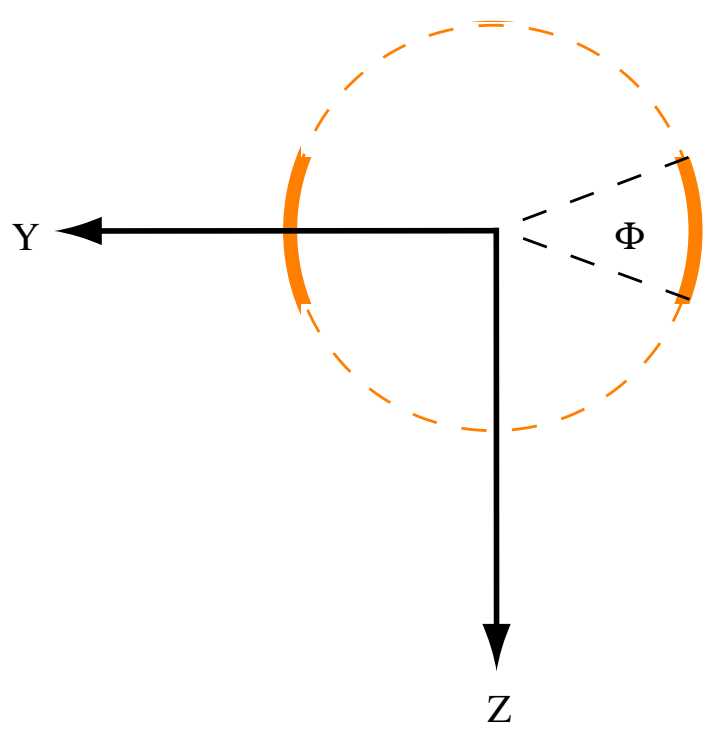

(c)

Figure 5: Quantifying the Size of the Magnetometer Measurement Locus Available For Estimation. 


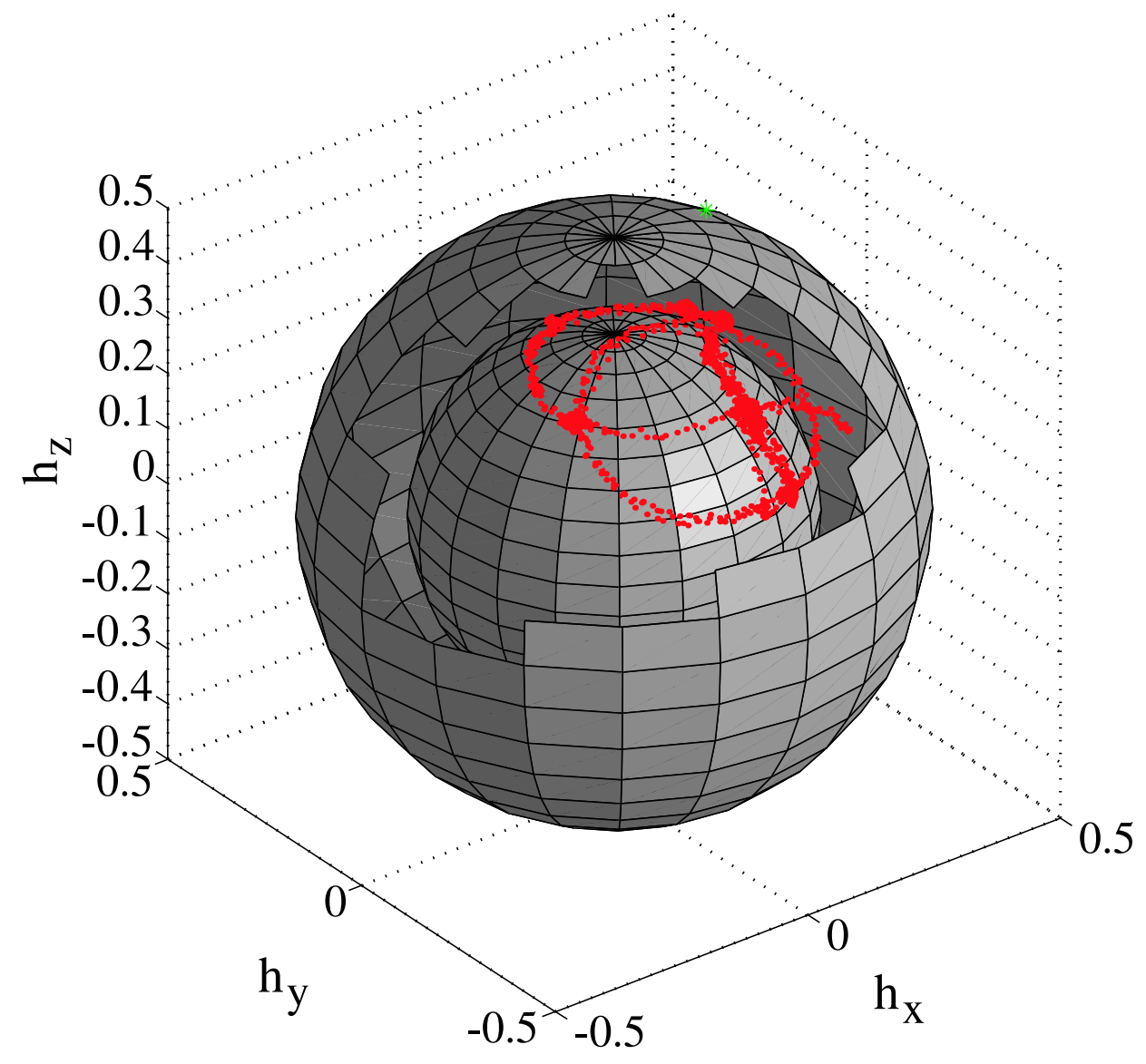

Figure 6: Portion of the Ellipsoid Representing the Locus of Magnetometer Measurements from Actual Experimental Data. The Actual Measurement Data is Shown by the Dark Color Dots on the Smaller (Inner) Sphere. After Calibration the Locus of Measurements will lie on the Larger Outer Sphere which has a Radius Equal to the Magnitude of the Local Magnetic Field Vector . 

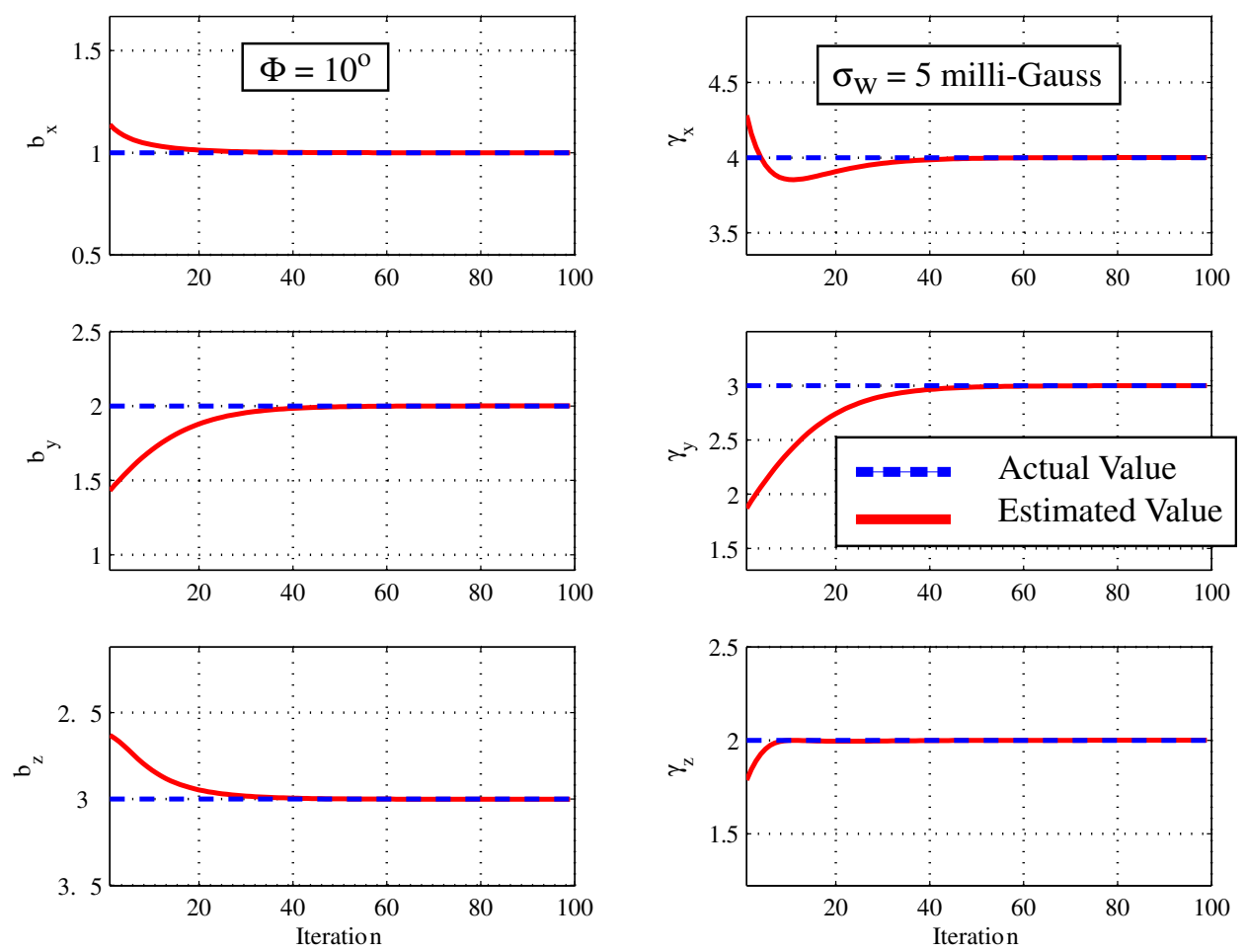

Figure 7: Hard Iron Caused Null-Shift (in Gauss) and and $\gamma$ (Unit-less) for the Iterative Least Squares Estimator. 

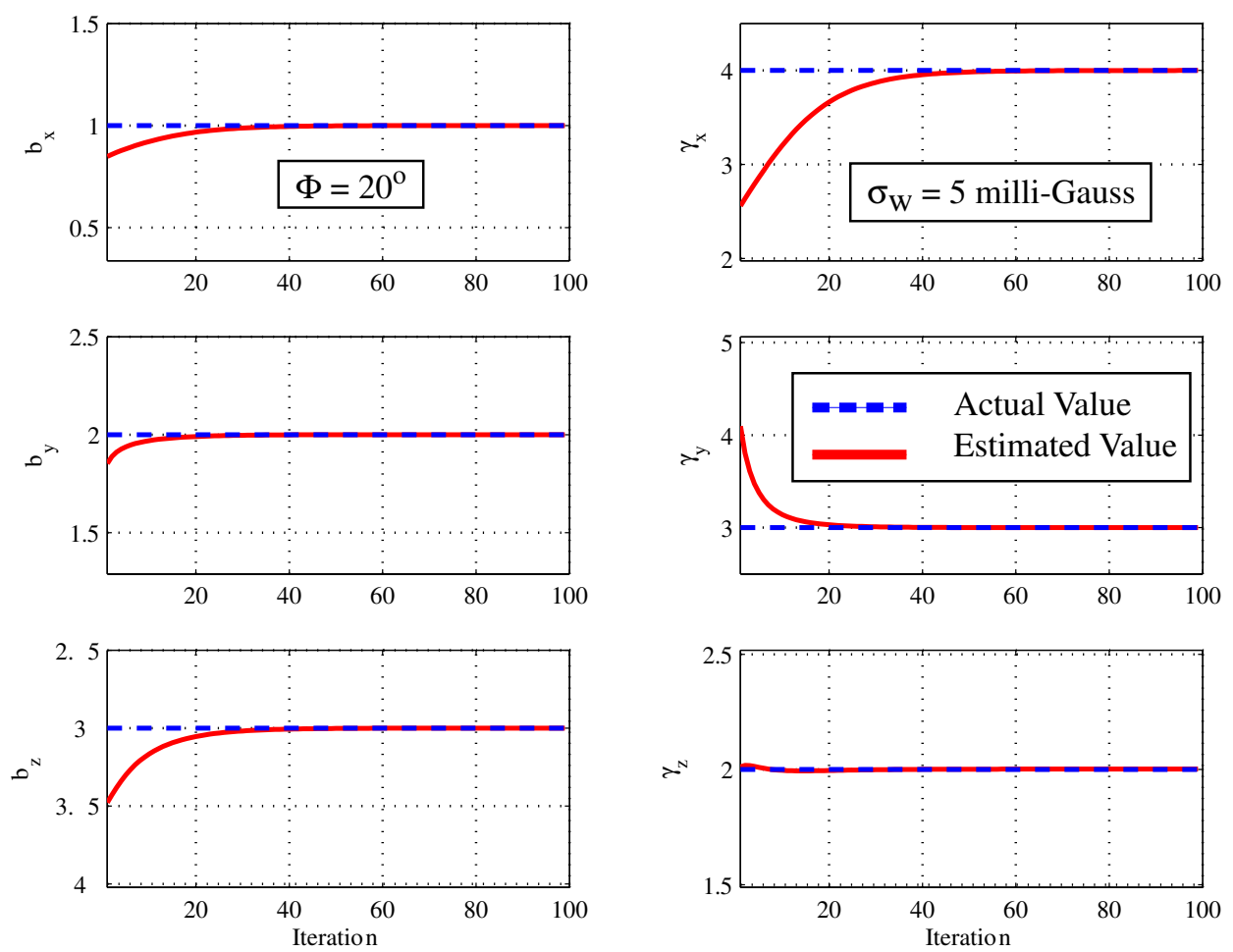

Figure 8: Hard Iron Caused Null-Shift (in Gauss) and and $\gamma$ (Unit-less) for the Iterative Least Squares Estimator. 

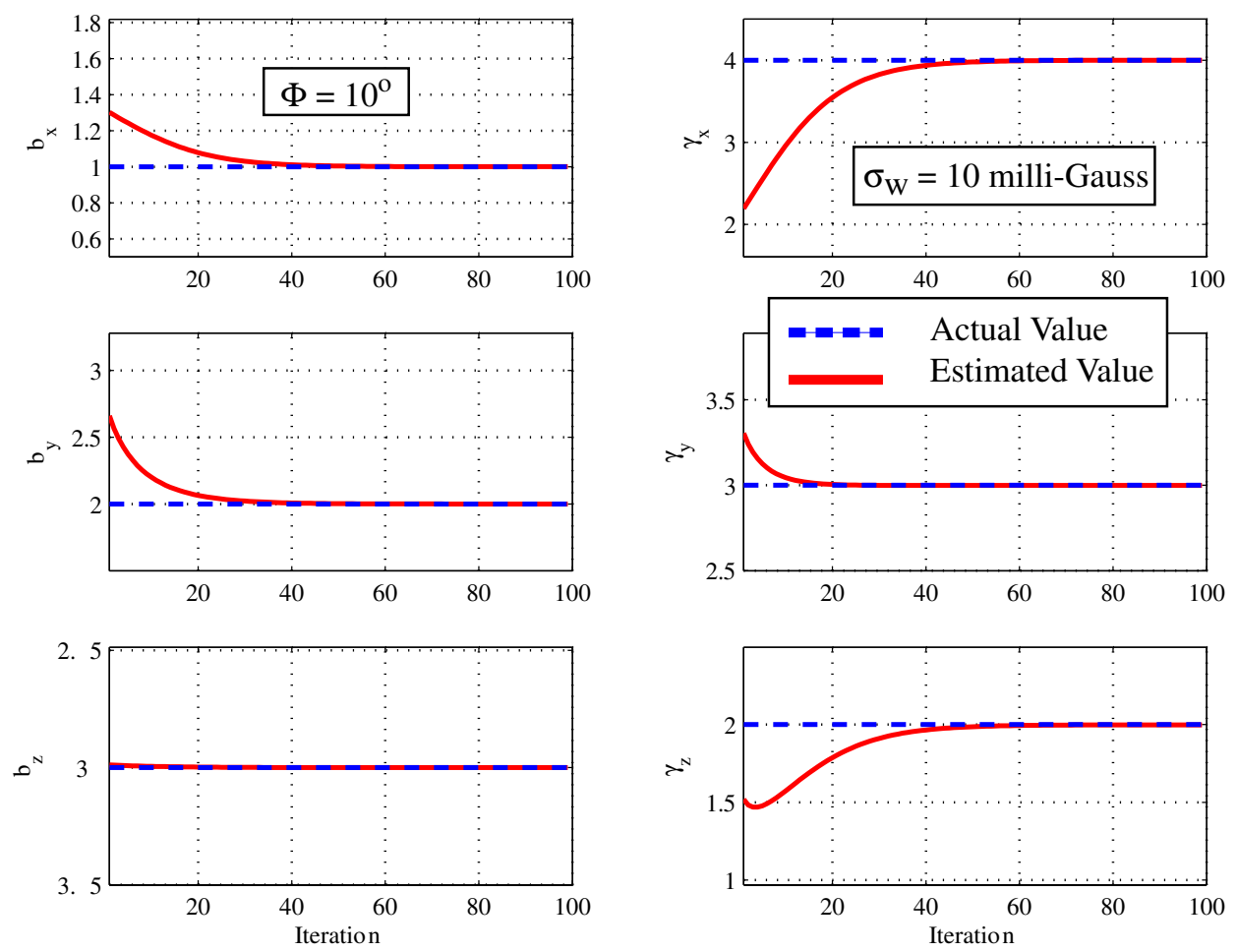

Figure 9: Hard Iron Caused Null-Shift (in Gauss) and and $\gamma$ (Unit-less) for the Iterative Least Squares Estimator. 

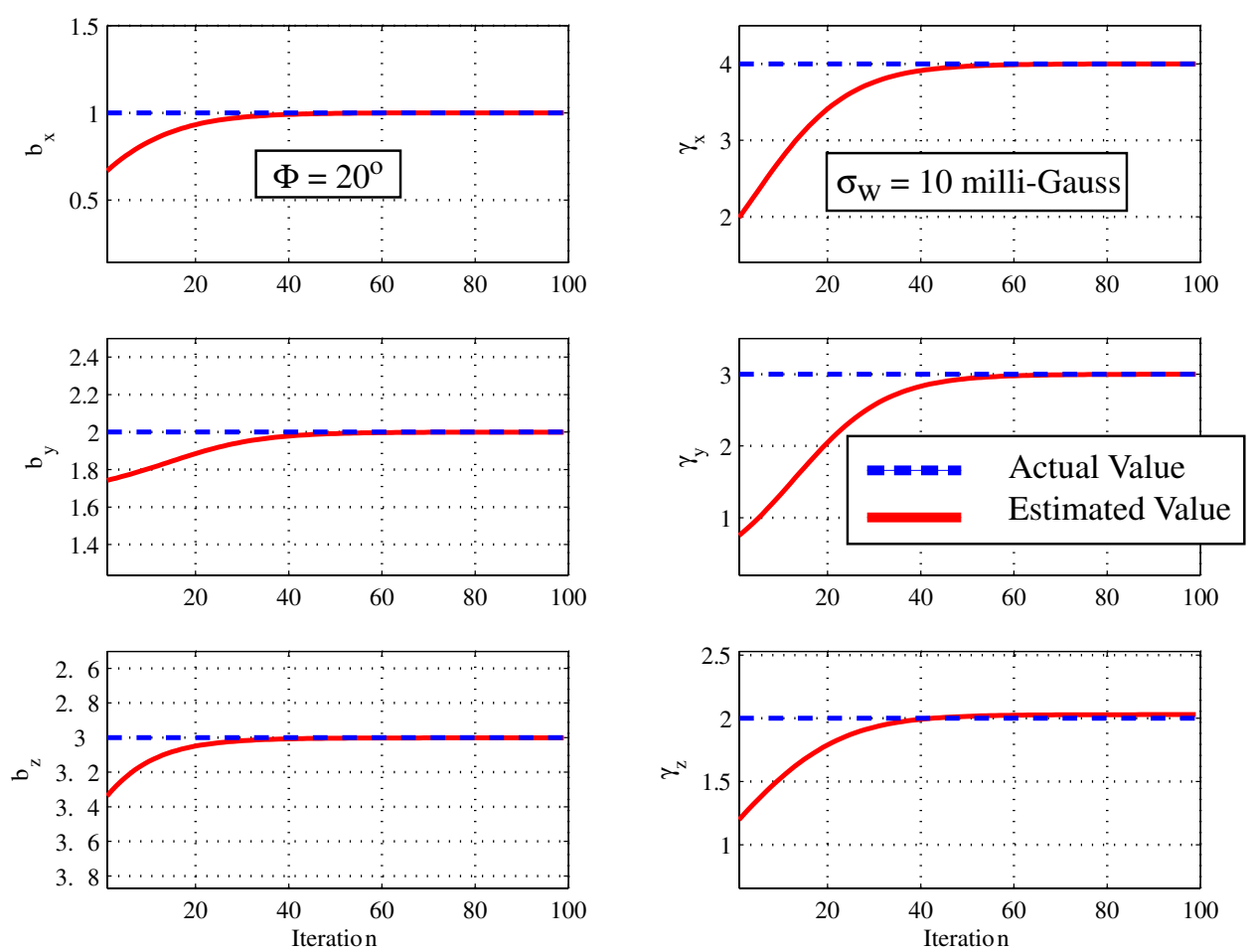

Figure 10: Hard Iron Caused Null-Shift (in Gauss) and and $\gamma$ (Unit-less) for the Iterative Least Squares Estimator. 

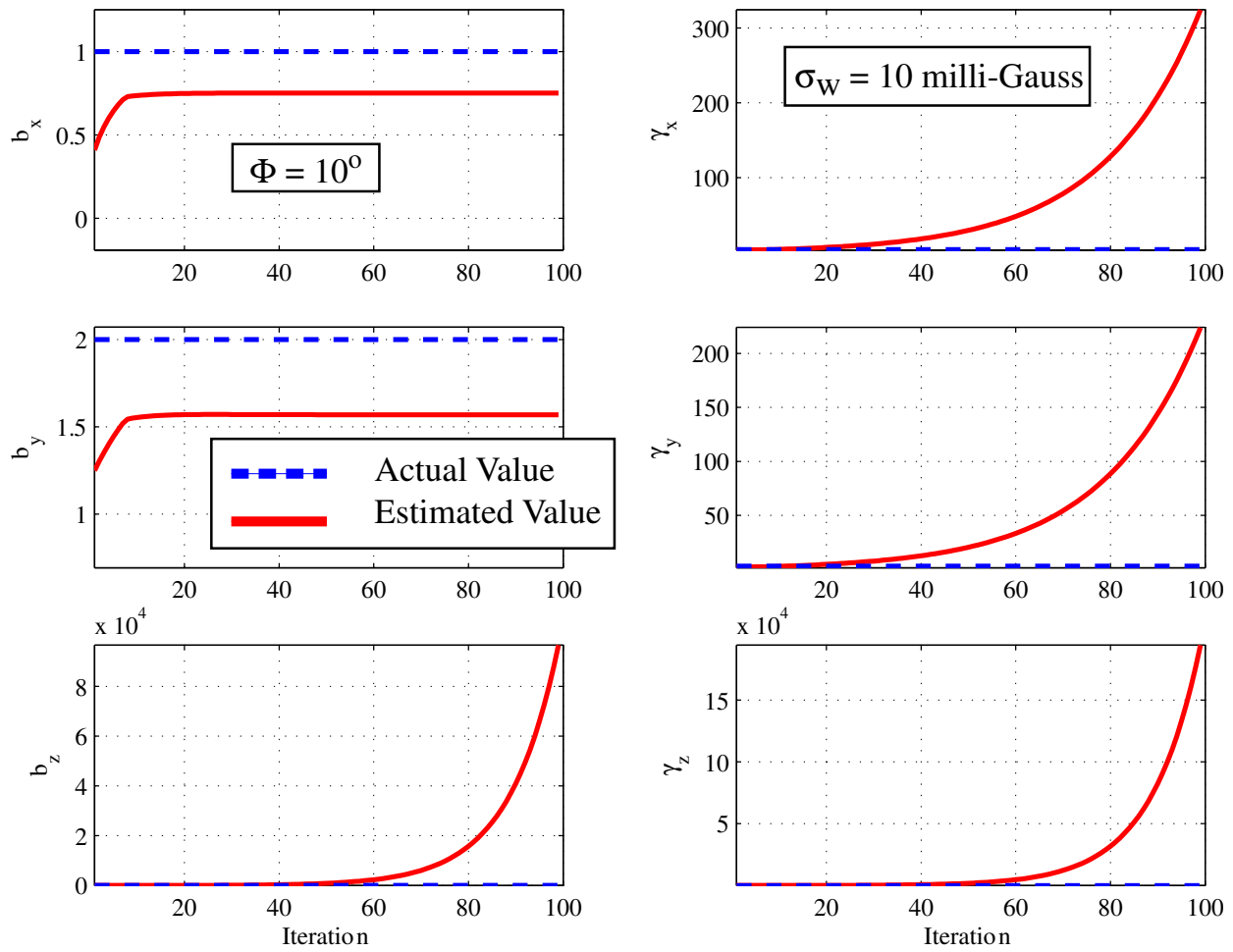

Figure 11: Divergence of the Hard Iron Caused Null-Shift (in Gauss) and and $\gamma$ (Unit-less) Estimates for the Iterative Least Squares Estimator. 

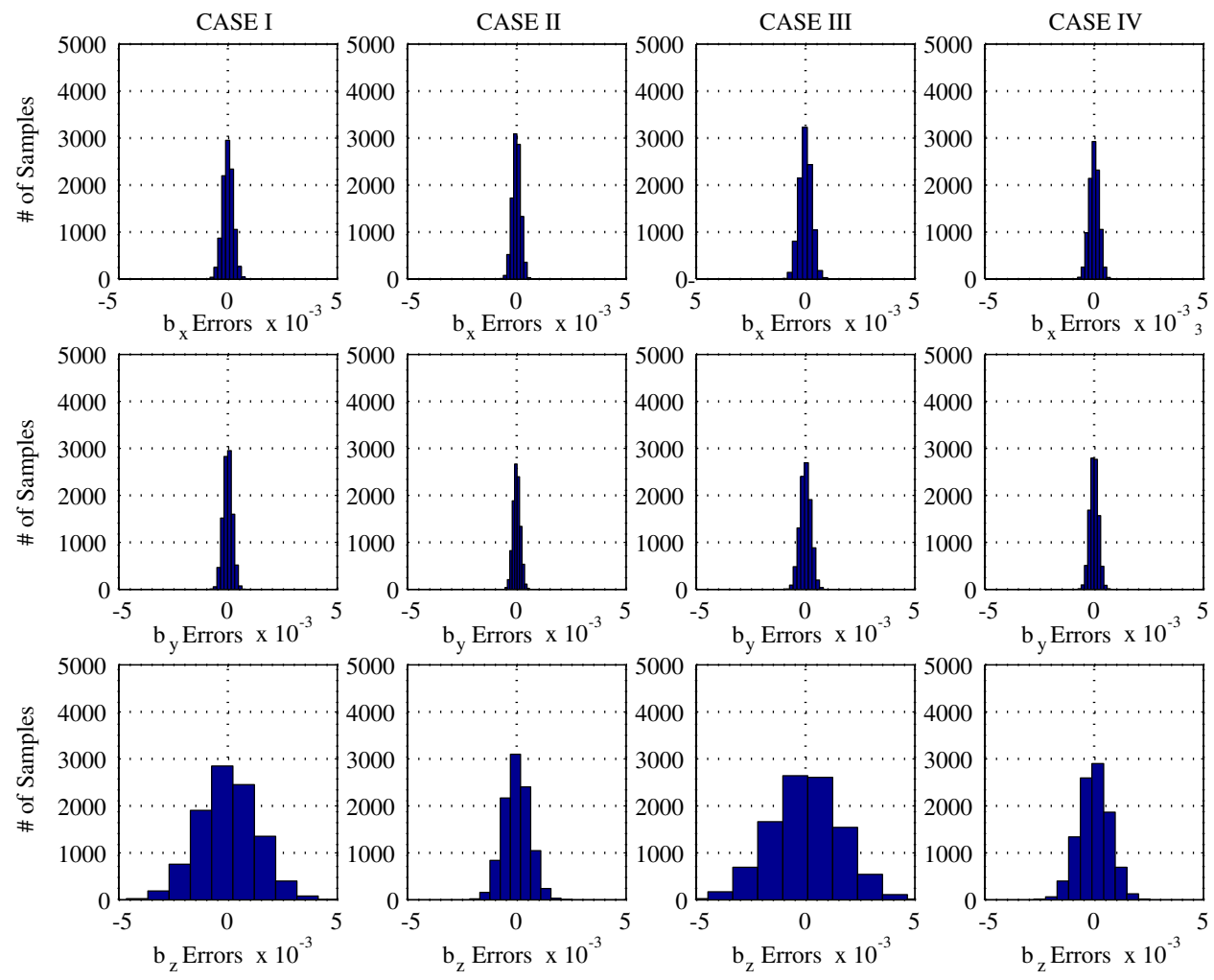

Figure 12: Hard Iron Bias Estimation Errors for the when using the Non-Linear, Two-Step Estimator for Initialization. Result from 10,000 Monte Carlo Runs . 

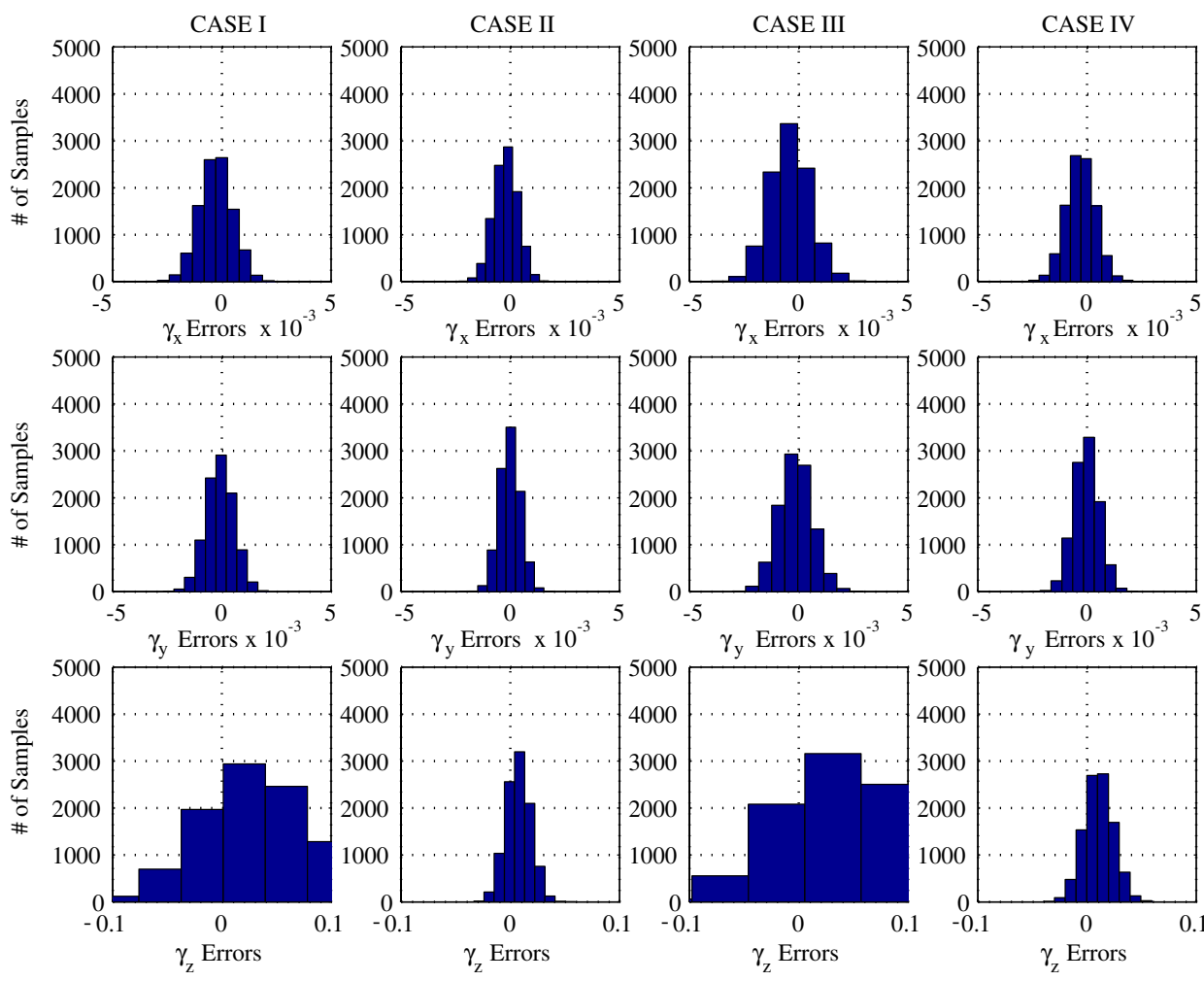

Figure 13: Scale Factor/Soft Iron $(\gamma)$ Estimation Errors when using the Non-Linear, Two-Step Estimator for Initialization. (Note: The Errors Are Unit-less). 


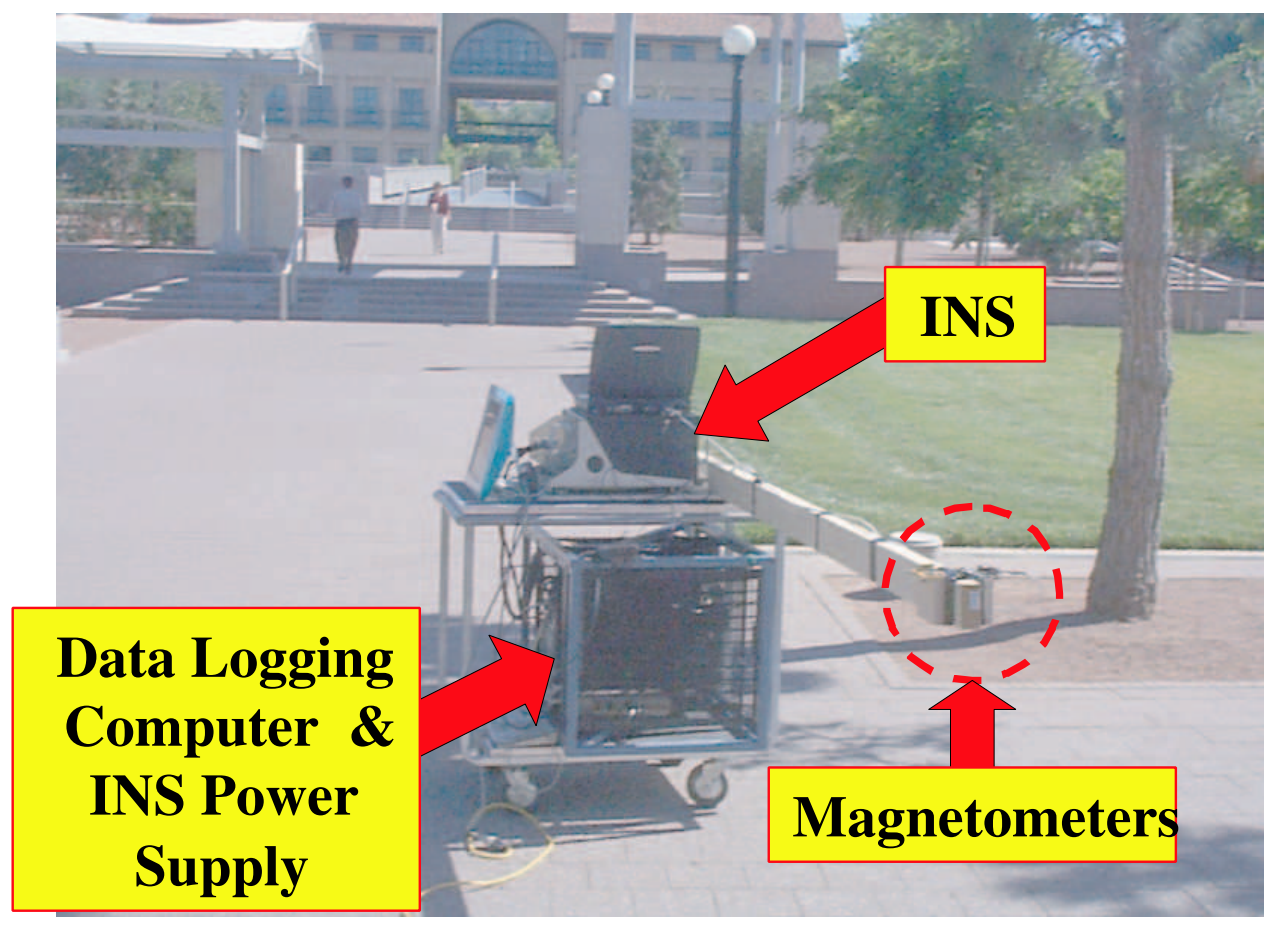

Figure 14: Experimental Setup for Ground Test. 

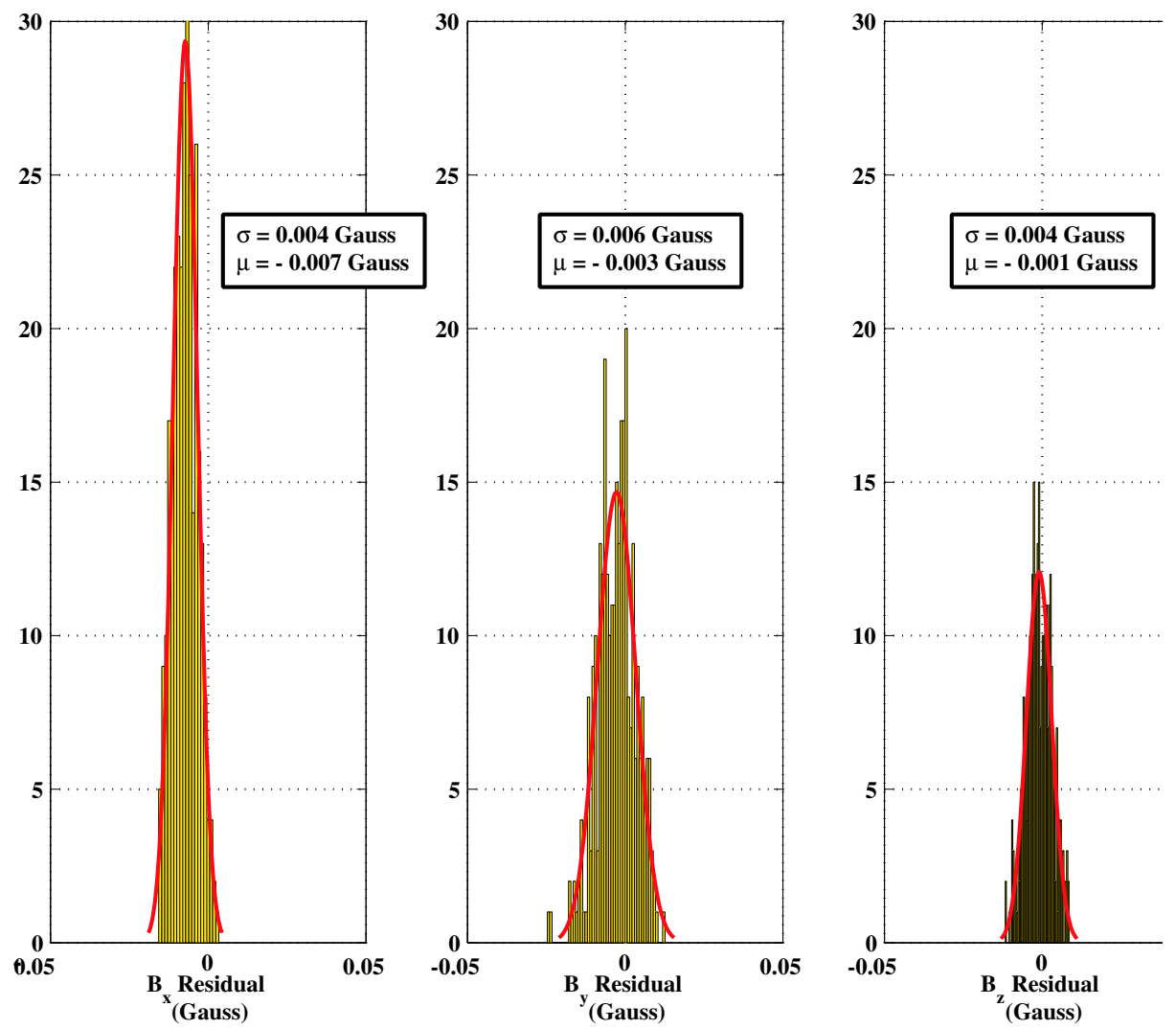

Figure 15: Magnetometer Calibration Residuals. 


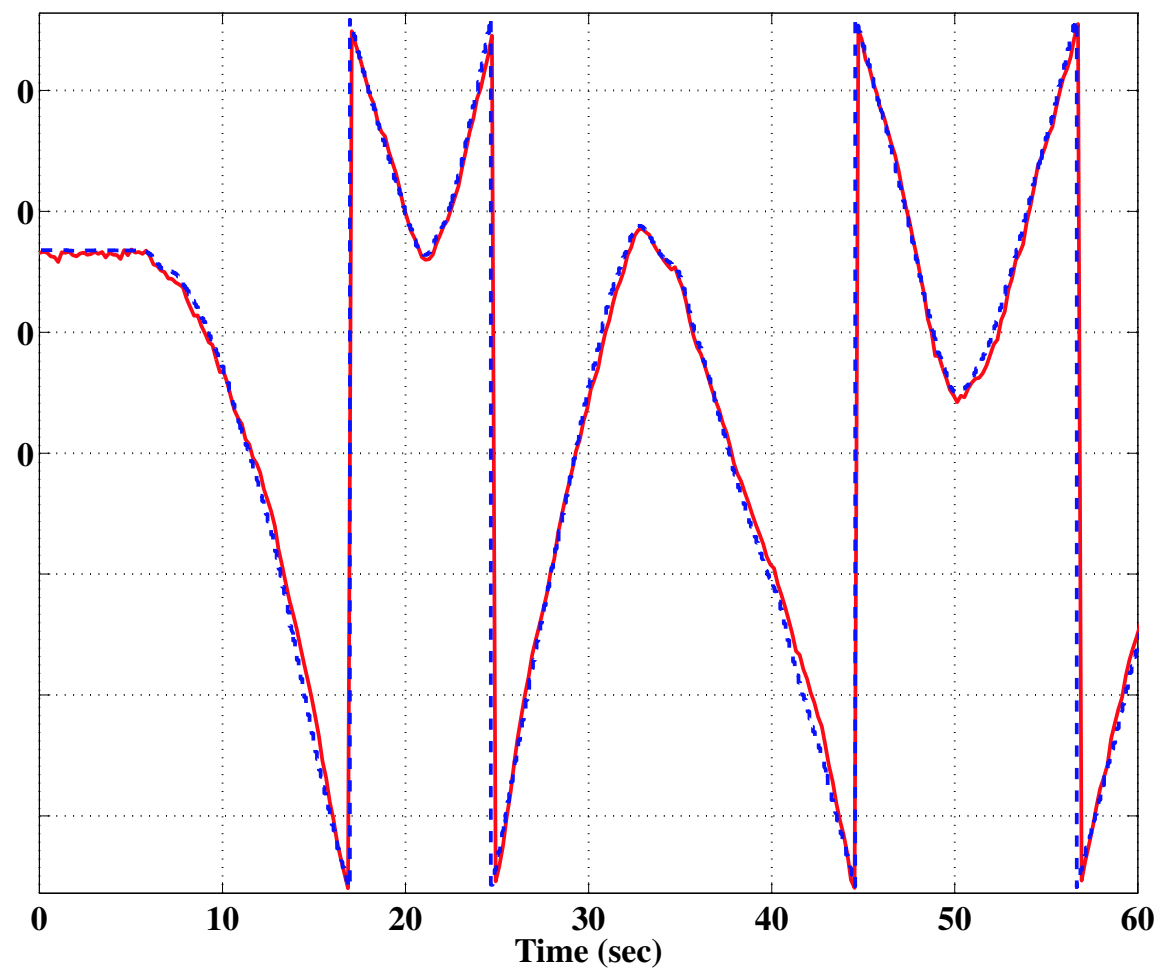

Figure 16: Comparison of INS Heading and Magnetometer Heading After Calibration. 


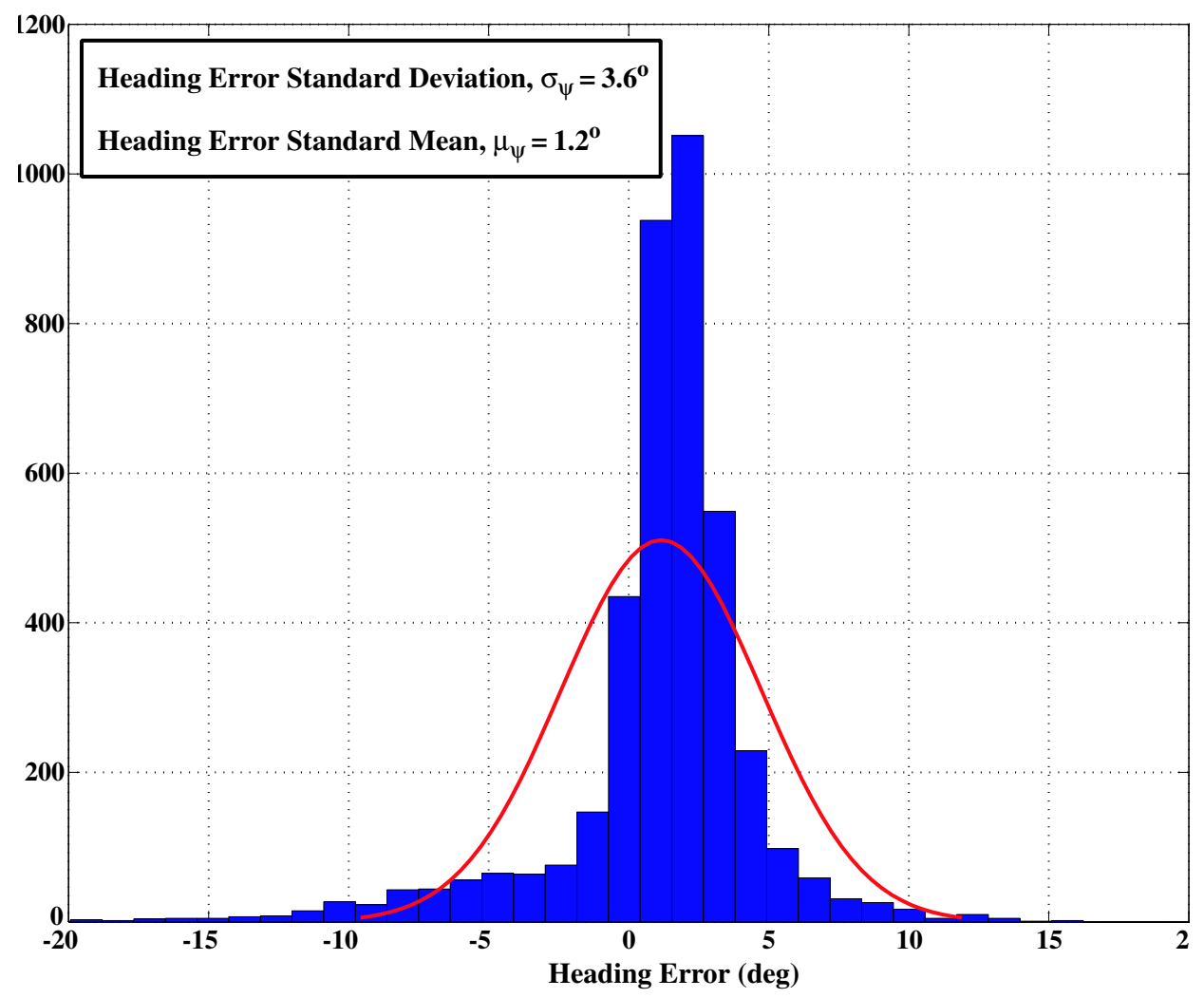

Figure 17: Histogram of Post Calibration Heading Errors. 